\title{
DISEÑO DE ESTRUCTURAS CON AISLAMIENTO SÍSMICO MEDIANTE EL USO DE ESPECTROS DE DISEÑO POR CAPACIDAD
}

\author{
Arturo Tena Colunga ${ }^{(1)}$
}

\begin{abstract}
RESUMEN
Se presenta el concepto de espectro de diseño por capacidad (EDC) para el diseño de estructuras aisladas sísmicamente, particularmente para estructuras cuyo sistema de aislamiento tiene un comportamiento histerético bilineal. Los EDC relacionan respuestas máximas no lineales de aceleración, velocidad y desplazamiento con el periodo efectivo del sistema aislado para sistemas bilineales definidos a partir de su fuerza de fluencia y de su pendiente posterior a la fluencia. Estos espectros no lineales de capacidad pueden ser muy útiles para intentar llegar a diseños óptimos de sistemas de aislamiento sísmico con comportamiento bilineal. El concepto de EDC y su potencial aplicación en el diseño de estructuras aisladas se ilustra con el estudio de dos estructuras específicas, donde el diseño del sistema de aislamiento se hizo utilizando los EDC y su eficacia se evaluó por medio de análisis dinámicos paso a paso no lineales del modelo ante la acción de componentes bidireccionales de los movimientos del terreno para una familia de acelerogramas asociados a los EDC. Los resultados de estos estudios sugieren que los EDC propuestos constituyen una herramienta útil y confiable para el diseño de estructuras aisladas sísmicamente con sistemas con comportamiento histerético bilineal. Sin embargo, se requieren continuar los esfuerzos de investigación que se han conducido en esta línea para definir EDC que puedan incorporarse en los reglamentos mexicanos de diseño sísmico.
\end{abstract}

\section{SUMMARY}

This paper presents the concept of inelastic capacity design spectra (ICDS) for the design of base-isolated structures; particularly those structures using isolators with a bilinear hysteretic behavior when subjected to dynamic loading. The inelastic capacity design spectra relate peak nonlinear accelerations, velocities, displacements and effective isolated natural periods for bilinear systems with a given yield strength and post yield stiffness. Inelastic capacity design spectra could be useful for the design of base isolators with bilinear hysteretic behavior, as these devices can be designed for a fixed yield strength and post yield stiffness. The concept of inelastic capacity design spectra and its application for the design of base isolated structures is illustrated with the case study of two structures. Nonlinear dynamic analyses conducted for the subject buildings suggest that the proposed ICDS are useful and reliable for the design of base-isolated structures, despite of the shortcomings of the present study, among them, using a limited strong ground motions data base and a crude statistical procedure to define the ICDS. Therefore, the concept of inelastic capacity design spectra seems to be a promising tool for reliable design of base isolators with bilinear hysteretic behavior. However, further research is needed in order to define ICDS for specific Mexican building codes.

Artículo recibido el 14 de agosto de 2000 y aprobado para su publicación el 4 de agosto de 2001. Se aceptarán comentarios y/o discusiones hasta cinco meses después de su publicación.

(1) Departamento de Materiales, Universidad Autónoma Metropolitana Azcapotzalco, Edificio H, 3er Piso, Av. San Pablo \# 180, Col. Reynosa Tamaulipas, 02200 México, DF. e-mail: atc@correo.azc.uam.mx 


\section{INTRODUCCIÓN}

En la última década el aislamiento de base ha emergido como una opción estructural en zonas sísmicas gracias al gran esfuerzo que durante los últimos treinta años ha realizado la comunidad estructural en todo el mundo, particularmente países como Nueva Zelanda, Estados Unidos y Japón. En este lapso de tiempo se han dedicado esfuerzos notables de investigación experimental y analítica para entender el comportamiento de diversos sistemas de aislamiento de base, que han permitido además, entre otras cosas, el desarrollo de prácticas de diseño para sistemas de aislamiento específicos, así como recomendaciones de diseño reglamentarias, tales como aquellas que se encuentran en el reglamentos UBC (Uniform Building Code) para edificios desde $1991 \mathrm{y}$ AASHTO (American Association of State Highway and Transportation Officials) para puentes desde 1990. De hecho, el nuevo reglamento de diseño nacional de los Estados Unidos, el International Building Code (IBC), en su primera versión del año 2000, ya incorpora directamente en sus normas de diseño por sismo los criterios de diseño de estructuras con aislamiento de base, que son muy similares a los del reglamento UBC-97, donde aún aparecían en un apéndice. En el Japón, el reglamento AIJ refiere desde 1986 a un documento que presenta recomendaciones para el diseño sísmico de estructuras con aislamiento de base, aunque estas disposiciones no tienen carácter legal ni su observación se hace de manera obligatoria.

El creciente interés en la aplicación de aislamiento sísmico en estructuras y puentes, tanto para diseño original como para proyectos de reparación, está directamente ligado a la aparición de recomendaciones de diseño sísmico en países como los Estados Unidos y Japón. En la figura 1 se presente un diagrama donde se censa el número de estructuras aisladas sísmicamente en algunos países de interés hasta 1990, mientras que en la figura 2 se presenta el censo hasta 1999.
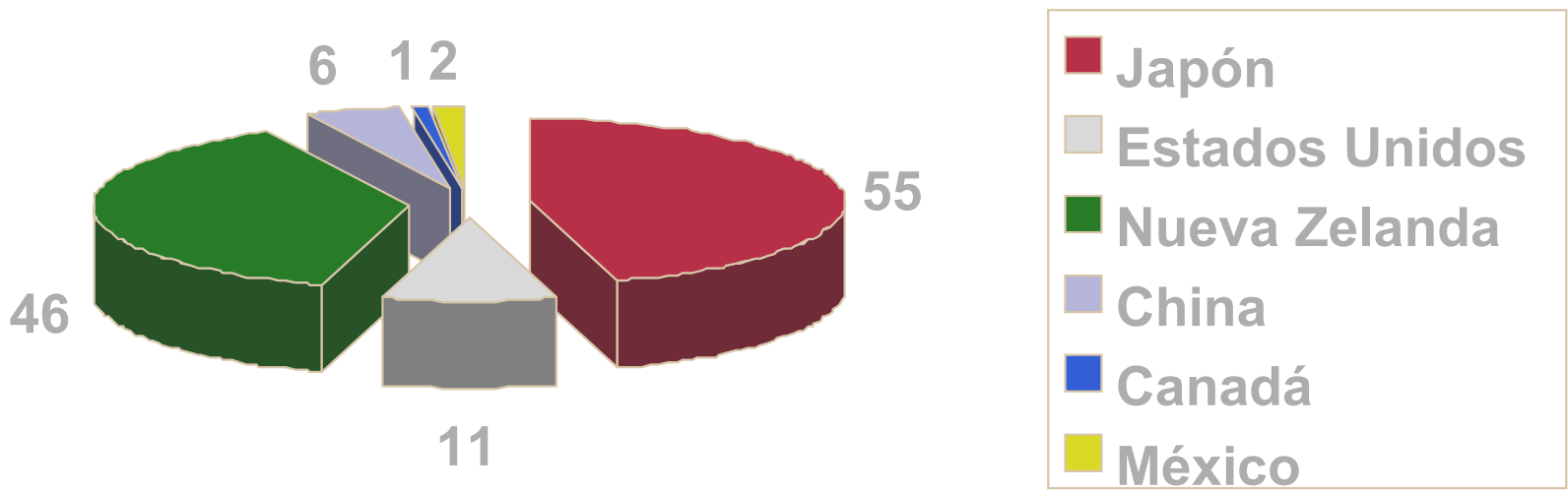

Figura 1. Edificios y puentes aislados sísmicamente en algunos países del mundo hasta 1990 
No es obra de la casualidad que el notable aumento de aplicaciones en países como los Estados Unidos y Japón coincida con la aparición de recomendaciones de diseño sísmico en sus reglamentos. Canadá también ha comenzado a verse beneficiada con la aparición y el desarrollo de normas y procedimientos de diseño de los Estados Unidos, ya que sus estructuras aisladas (puentes) son proyectos con sistemas desarrollados y patentados en los Estados Unidos, cuyo diseño se hizo observando recomendaciones de aquel país. En China se observa un fenómeno interesante fruto de la cooperación internacional de un proyecto multidisciplinario auspiciado por la Organización de Desarrollo Industrial de las Naciones Unidas (UNIDO), sin que el aumento de aplicaciones se deba a la aparición de una normatividad en ese país. Nueva Zelanda es el país que primero contó con recomendaciones de diseño, particularmente para los sistemas que ahí se desarrollaron, y eso se refleja en que hasta 1990 mantenían el liderato de aplicaciones en el ámbito mundial (principalmente puentes); sin embargo, el número de aplicaciones en la última década ha disminuido apreciablemente, tal vez porque siendo un país poco poblado ha requerido de poca ampliación de su infraestructura carretera en esta década. En México las aplicaciones se han dado principalmente por la intervención directa de los desarrolladores de las tecnologías nacionales: el Ing. Manuel González Flores y su sistema deslizante a base de balines metálicos que se aplicó a un par de estructuras en la década de los años setenta, y el Ing. Federico Garza Tamez y su sistema pendular GT-BIS, cuyas aplicaciones se han hecho durante la segunda mitad de la década de los años noventa.
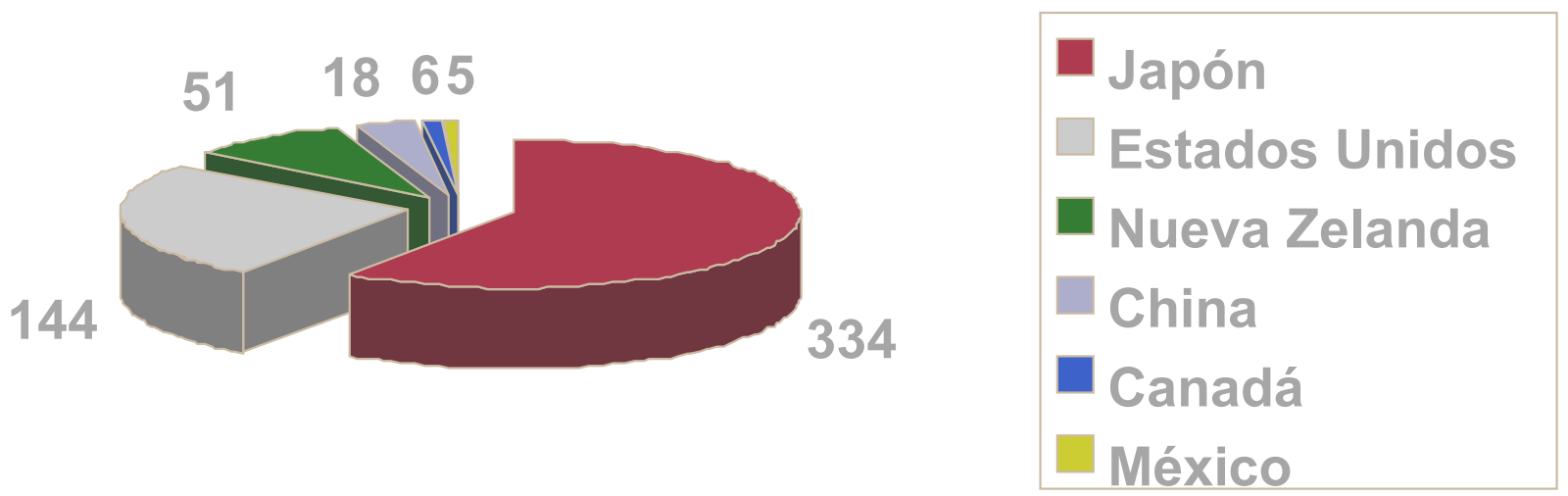

Figura 2. Edificios y puentes aislados sísmicamente en algunos países del mundo hasta 1999 
De la figura 2 se observa que el liderato mundial en cuanto aplicaciones lo tiene actualmente Japón, en parte porque sus recomendaciones son menos restrictivas y no hacen prohibitiva la aplicación de aislamiento sísmico, como en opinión de algunos expertos lo hacen algunas de las disposiciones del reglamento UBC (Kelly, 1999).

Por lo general, el diseño sísmico de aisladores de base está directamente asociado por el desplazamiento dinámico máximo que debe permitirse a los aisladores por concepto de estabilidad más que por algún criterio de resistencia. Aunque este hecho ha sido reconocido en la literatura especializada desde hace tiempo, las prácticas de diseño propuestas por los reglamentos de los Estados Unidos (por ejemplo UBC-97 e IBC-2000) se basa en un procedimiento donde el desplazamiento se diseño se obtiene indirectamente a partir de su espectro de diseño de pseudoaceleraciones especificado para el diseño de estructuras convencionales.

Un diseño óptimo de estructuras con aislamiento sísmico requiere tener, desde el punto de vista espectral, toda la información relevante a la mano. Los espectros de aceleraciones solamente proporcionan una tercera parte de la información requerida, ya que las respuestas máximas de velocidad y de desplazamiento no se incluyen. Los espectros de aceleraciones son importantes para el diseño de la superestructura; sin embargo, para el diseño del sistema de aislamiento es más relevante el espectro de desplazamientos. Por otra parte si el espectro de aceleraciones seleccionado es una envolvente de diseño que incluye mesetas y caídas para periodos largos que toman en cuenta incertidumbres en varios parámetros relevantes para el diseño de estructuras convencionales, entonces los desplazamientos asociados a dicho espectro dejan de ser rigurosamente proporcionales $\mathrm{y}$, por tanto, un espectro de diseño de desplazamiento obtenidos a partir de un espectro de diseño de aceleraciones puede tener poco sentido para el diseño de sistemas de aislamiento. Esto ha sido reconocido por el autor y sus colaboradores, y se han dado los primeros pasos para definir, con fines reglamentarios, ecuaciones para obtener desplazamientos de diseño en sistemas de aislamiento sísmico a partir de espectros de desplazamiento (por ejemplo, Villegas 1999, Villegas y Tena 1999, y Villegas-Jiménez y TenaColunga 2000).

Sin embargo, si se desea optimar el proceso de diseño, se requieren de espectros donde las respuestas máximas de desplazamiento, velocidad y aceleración sean incluidas directamente. Desde ese punto de vista, los espectros tripartitos de diseño inelástico (ETDI) propuestos por Newmark y Hall (1982) son potencialmente más útiles, ya que la respuesta no lineal de estructuras con aislamiento sísmico se concentra en el sistema de aislamiento, y este tipo de espectros proporciona toda la información que se requiere, ya que incluyen las respuestas máximas de aceleración, velocidad y desplazamiento. Sin embargo, los ETDI que propusieron Newmark y Hall se basan en el concepto de demandas de ductilidad por desplazamiento constantes, que en opinión del autor no es el mejor concepto para el diseño de sistemas de aislamiento. Para un acelerograma dado, una demanda de ductilidad por desplazamiento fija puede estar asociada a distintos cortantes de fluencia (figura 3), independientemente del tipo de comportamiento inelástico (elasto-plástico perfecto, bilineal, etc), es decir, esta demanda de ductilidad por desplazamiento no es única, como lo han ilustrado antes, entre otros autores, Miranda (1993). 


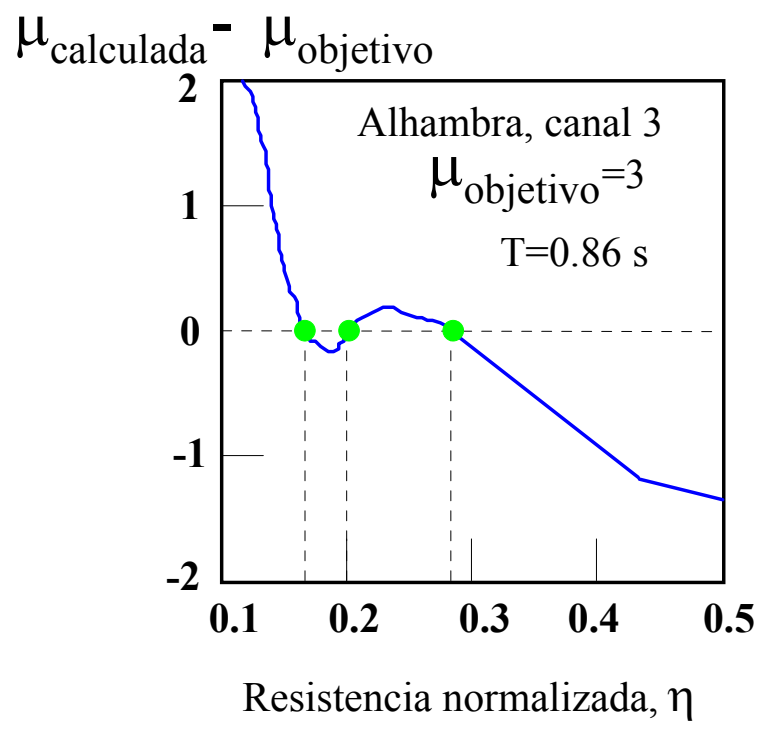

Figura 3. Ejemplo de distintos cortantes de fluencia asociados a una misma demanda de ductilidad (adaptado de Miranda, 1993)

Los sistemas de aislamiento sísmico, particularmente aquéllos con comportamiento histerético de tipo bilineal (como es el caso de la mayoría de los sistemas a base de elastómeros), pueden diseñarse con base en una fuerza de fluencia y pendiente posterior a la fluencia previamente establecidas. Por lo tanto, desarrollar lo que el autor define como espectro de capacidad (EC) en una gráfica tetralogarítmica puede ser más útil para el diseño de sistemas de aislamiento con estas características. Los EC relacionan respuestas máximas no lineales de aceleración, velocidad y desplazamiento con el periodo efectivo del sistema de aislamiento para sistemas bilineales definidos a partir de su fuerza de fluencia (o cedencia) y de su pendiente posterior a la fluencia. Estos espectros no lineales de capacidad pueden ser muy útiles para intentar llegar a diseños óptimos de sistemas de aislamiento sísmico con comportamiento bilineal, ya que en general, en la práctica mundial se intenta, en lo posible, diseñar a los sistemas de aislamiento para la menor fuerza de fluencia que brinde un nivel de seguridad razonable o requerido, ya que el costo de muchos sistemas de aislamiento se incrementa a medida que aumenta su fuerza de fluencia de diseño, particularmente para sistemas elastoméricos. El concepto de EC en gráfica tetralogarítmica y su potencial aplicación para el diseño de sistemas de aislamiento sísmico se presenta y discute en las siguientes secciones.

\section{CONCEPTO DE ESPECTRO DE DISEÑO POR CAPACIDAD}

El espectro de diseño por capacidad (EDC) en gráfica tetralogarítmica es un concepto que propone el autor para facilitar el diseño sísmico de estructuras aisladas en su base utilizando un análisis espectral, ya que la acción no lineal de estructuras aisladas sísmicamente se concentra precisamente en el sistema de aislamiento. Estos espectros de diseño son, en realidad, envolventes de espectros no lineales de capacidad que pueden calcularse para distintos sistemas de aislamiento. La utilización de estos espectros para el diseño simplificado de aisladores de base puede conducir a diseños iniciales más confiables que utilizando un espectro de diseño de aceleraciones, como lo considera actualmente el reglamento UBC, e incluso, puede limitar los 
casos donde se requieran realizar forzosamente análisis dinámicos paso a paso para el diseño de estructuras aisladas en su base, por las razones que se enuncian a continuación.

Los espectros de diseño por capacidad en gráfica tetralogarítmica se construyen a partir de espectros no lineales de capacidad para cada tipo de aislador, tomando en cuenta una familia de acelerogramas dada. Estos espectros no lineales de capacidad consisten en calcular el espectro no lineal de respuesta de sistemas que tiene un cortante de fluencia y un modelo de histéresis definido (por ejemplo, un modelo bilineal); es decir, difieren de los espectros de respuesta inelásticos propuestos por Newmark y Hall (1982) en que, mientras que en estos últimos se tiene fijo el valor de ductilidad por desplazamiento (por ejemplo, $\mu=2$ ) para el modelo de histéresis seleccionado, en los espectros propuestos lo que se fija es el cortante de fluencia (por ejemplo, $\mathrm{V} / \mathrm{W}=0.10)$.

Estos espectros relacionan respuestas máximas no lineales de aceleración, velocidad y desplazamiento con periodos efectivos del sistema aislado con cortantes de fluencia y pendientes posteriores a la fluencia conocida. Esta variante resulta más propia para sistemas de aislamiento, los cuales pueden diseñarse con base en su cortante de cedencia y pendiente posterior a la fluencia, más que en conceptos de ductilidad global.

Es un hecho que los principales sistemas de aislamiento se diseñan para un cortante de fluencia y pendiente posteriores a la fluencia conocidos y que, generalmente, el diseño final de los aisladores de base es controlado por el desplazamiento máximo admisible del aislador para estabilidad dinámica más que por su resistencia a carga lateral, por lo que el uso de los espectros de capacidad en gráfica tetralogarítmica puede resultar muy útil para definir el desplazamiento no lineal máximo esperado para una estructura dada y, en función a ello, definir cuáles son las características más deseables de los aisladores para lograr un aislamiento sísmico óptimo y eficaz. En las secciones siguientes se explicará e ilustrará el cálculo y la definición del espectro de capacidad.

\section{CÁLCULO DE LOS ESPECTROS DE CAPACIDAD}

El espectro de diseño por capacidad (EDC) para sistemas con aislamiento sísmico se define como la envolvente de los espectros de capacidad calculados a partir de sistemas de un grado de libertad que representan sistemas de aislamiento con características histeréticas globales definidas y para una base de datos de acelerogramas que resulten representativos para definir un espectro de diseño base para una región con una sismicidad conocida o supuesta. Por lo tanto, para definir dichos espectros, fue necesario desarrollar un programa de cómputo que facilitara su cálculo, así como seleccionar un criterio de diseño basado en la estadística. El programa para calcular la respuesta dinámica de sistemas de un grado de libertad elásticas e inelásticas se calibró tanto con soluciones elásticas conocidas (espectros de respuesta elásticos) como con soluciones inelásticas conocidas (cálculo de soluciones no lineales para problemas reportados en la literatura).

Los EDC pueden definirse para cualquier modelo histerético de interés; sin embargo, dado que los sistemas de aislamiento que más se están utilizando en el mundo son a base de elastómeros, se decidió definir los espectros de capacidad para sistemas bilineales con pendientes 
posteriores a la fluencia del $10 \%$ de su rigidez elástica $\left(\mathrm{k}_{2} / \mathrm{k}_{1}=0.10\right)$, como se ilustra en la figura 4, ya que éste es un valor característico para aisladores de base de tipo elastomérico.

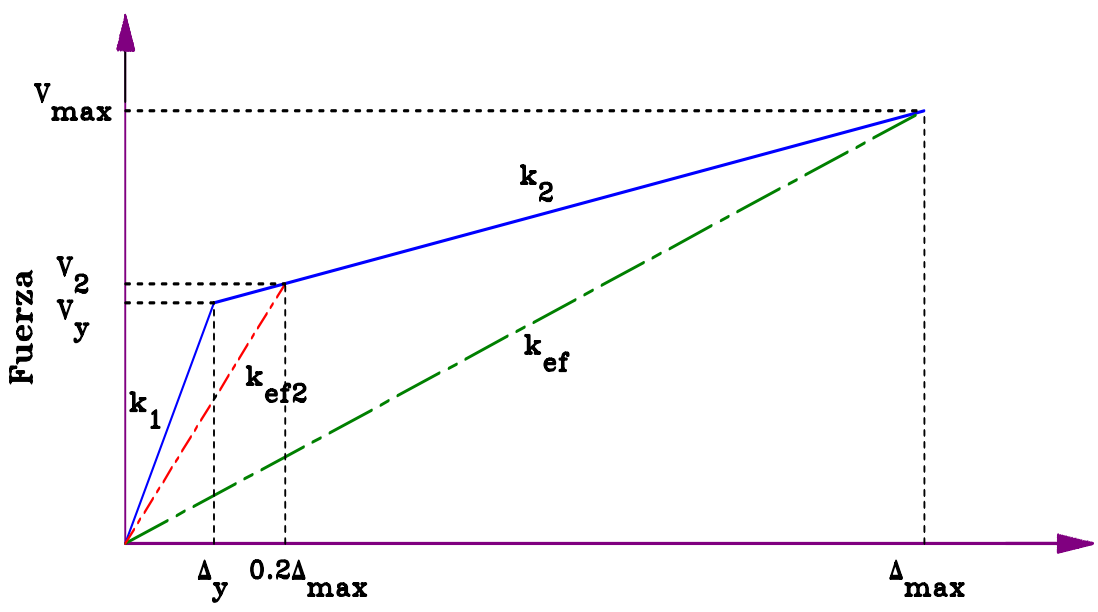

Deformación

Figura 4. Gráfica fuerza-desplazamiento para un aislador bilineal (propuesta mecánica del reglamento UBC)

El cálculo de espectros de capacidad para un acelerograma dado involucra los siguientes pasos:

(1) Definición los parámetros del modelo histerético seleccionado. Para un sistema bilineal, se define el cortante de fluencia normalizado con el peso total de la estructura aislada $\left(\mathrm{V}_{\mathrm{y}} / \mathrm{W}\right)$ y la relación entre la pendiente posterior a la fluencia con la pendiente elástica $\left(\mathrm{k}_{2} / \mathrm{k}_{1}\right)$.

(2) Selección del amortiguamiento viscoso equivalente $(\zeta)$ de referencia para la superestructura (típicamente $\zeta=0.05$ ), selección del intervalo de periodos de interés para los que se construirá el espectro, en función de un periodo inicial $\left(\mathrm{T}_{\mathrm{i}}\right)$ y uno final $\left(\mathrm{T}_{\mathrm{f}}\right)$, así como el incremento de periodos $(\Delta \mathrm{T})$ dentro del intervalo para el cálculo de respuestas dinámicas. Los incrementos pueden hacerse en escalas aritméticas o logarítmicas. Se recomienda utilizar incrementos logarítmicos, dado que los espectros se grafican en escala tetralogarítmica.

(3) Propuesta del periodo de interés $T_{j}$, donde $T_{j}=T_{j-1}+\Delta T, T_{i} \leq T_{j} \leq T_{f}$

(4) Para cada periodo de interés $T_{j}$ :

(a) Determinar que el paso de integración $(\Delta t)$ seleccionado para el acelerograma es adecuado para el cálculo de respuestas no lineales. Una regla que se ha propuesto en la literatura desde hace tiempo y que funciona razonablemente bien es revisar que $\Delta \mathrm{t} \leq \mathrm{T}_{\mathrm{j}} / 10$. En caso contrario, se requerirá interpolar el acelerograma en estudio. Se recomienda utilizar un algoritmo de interpolación selectivo, ya que no se requieren de pasos de integración pequeños para periodos relativamente largos, como si pueden requerirse para periodos cortos, cercanos a cero. 
(b) Para cada paso de integración $(\Delta t)$, utilizando un método numérico confiable (por ejemplo, el método Newmark- $\beta$ ), resolver la ecuación de movimiento de un sistema de un grado de libertad no lineal dada por:

$$
m \ddot{x}(t)+c(t) \dot{x}(t)+k(t) x(t)=F(t)
$$

donde $m, c(t)$ y $k(t)$ son respectivamente la masa, el coeficiente de amortiguamiento (al tiempo $t$ ) y la rigidez (al tiempo $t$ ) del sistema asociadas a $\mathrm{T}_{\mathrm{j}}, F(t)$ es la fuerza efectiva al tiempo $t, \quad \mathrm{y} \quad \ddot{x}(t), \dot{x}(t) y x(t)$ son respectivamente la aceleración, velocidad $\mathrm{y}$ desplazamiento al tiempo $t$, como se define en la literatura técnica sobre este tema.

(c) Guardar las respuestas máximas de aceleración, velocidad, desplazamiento y la fuerza restitutiva no lineal del sistema.

(d) Calcular el periodo efectivo del sistema aislado, $T_{a}$, a partir de:

$$
T_{a}=2 \pi \sqrt{\frac{m}{k_{e f}} ; \quad k_{e f}=\frac{F_{j \max }^{-}+F_{j \max }^{+}}{\Delta_{j \max }^{-}+\Delta_{j \max }^{+}}}
$$

donde $F_{\operatorname{maxj}}^{-} F_{\operatorname{maxj}}^{+}$y $\Delta_{\operatorname{maxj}}^{-} \Delta_{\operatorname{maxj}}^{+}$son respectivamente las fuerzas máximas y los desplazamientos máximos negativos y positivos y $k_{e f}$ es la rigidez efectiva del sistema no lineal de un grado de libertad con un periodo elástico inicial $\mathrm{T}_{\mathrm{j}}$. La definición de la rigidez efectiva $k_{e f}$ es ya un estándar en la literatura especializada sobre aislamiento sísmico (por ejemplo, Naeim y Kelly, 1999), incluyendo al reglamento UBC.

(5) Regresar al paso 3 y continuar hasta que $\mathrm{T}_{\mathrm{j}}=\mathrm{T}_{\mathrm{f}}$.

El cálculo y definición de los espectros de capacidad se ilustra con los pasos tomados para definir este espectro para un sistema bilineal con $\mathrm{V}_{\mathrm{y}} / \mathrm{W}=0.10$ y $\mathrm{k}_{2} / \mathrm{k}_{1}=0.10$. En la figura $5 \mathrm{se}$ presentan las respuestas no lineales máximas calculadas para este sistema cuando se sujeta al acelerograma ZACA-EW del sismo del 19/09/85. Con línea punteada se presenta la respuesta máxima de aceleraciones, con línea continua se indica la respuesta máxima de velocidades y con línea con rayas se presenta la respuesta máxima de desplazamientos. De esta gráfica se observa que las respuestas máximas no están directamente relacionadas entre sí, como consecuencia de tratarse de un sistema inelástico, y que existen zonas donde la respuesta no lineal está controlada por los desplazamientos, pero en otras controla la velocidad, como también lo han ilustrado previamente Newmark y Hall (1982). De esta figura se aprecia que, para un periodo cercano a cero $(\mathrm{T}=0.0)$, la aceleración máxima se aproxima a la aceleración máxima del terreno (línea punteada) y que el desplazamiento tiende a cero (línea con rayas), como debería esperarse aun para el caso de sistemas inelásticos. 


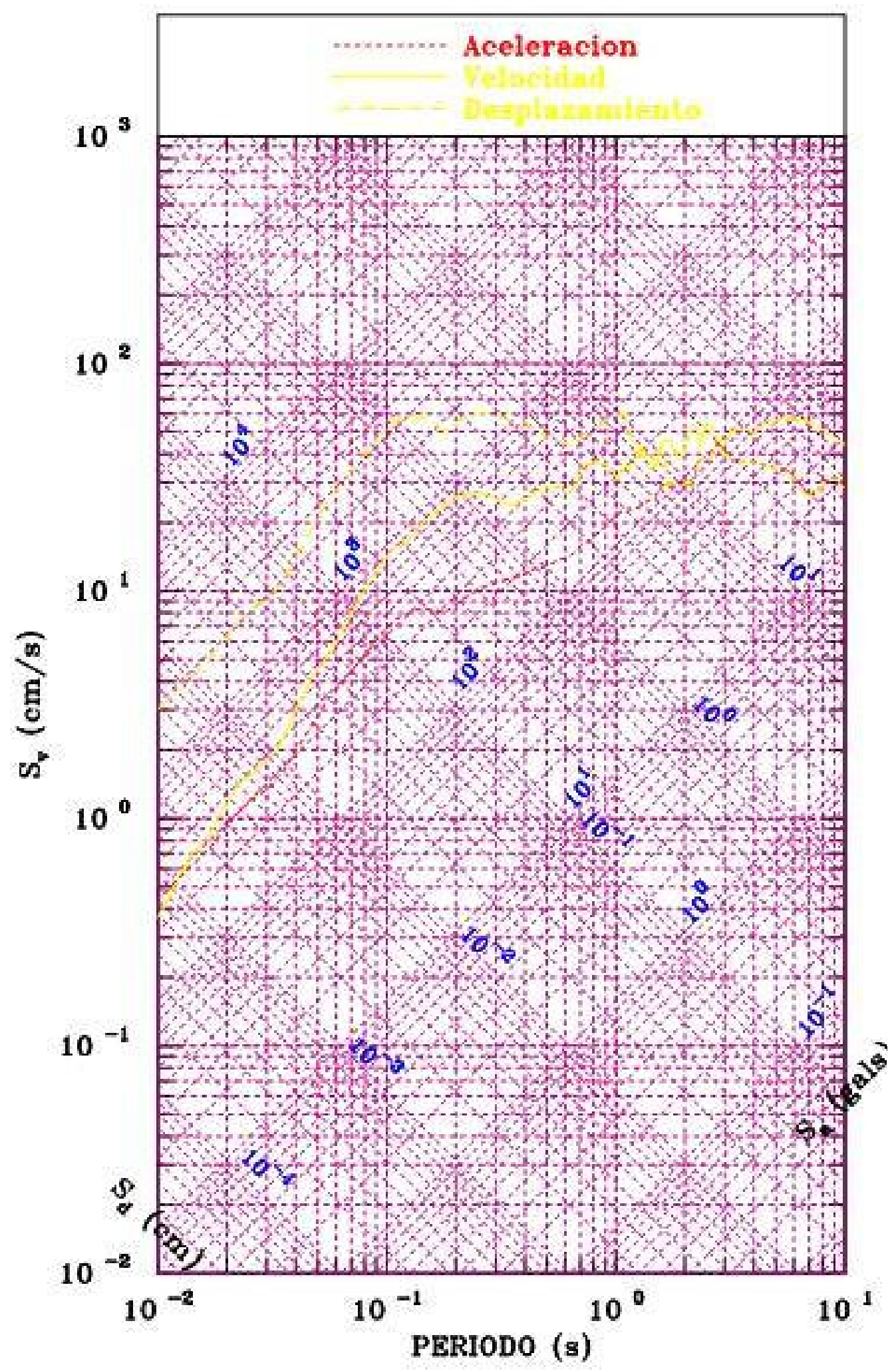

Figura 5. Espectro inelástico de capacidad para el acelerograma ZACA-EW del sismo del $19 / 09 / 85$ para elastómeros bilineales con parámetros $\mathrm{V}_{\mathrm{y}} / \mathrm{W}=0.10$ y $\mathrm{k}_{2} / \mathrm{k}_{1}=0.10$. 


\section{ESPECTROS DE DISEÑO POR CAPACIDAD}

Para definir espectros de diseño por capacidad (EDC) en gráfica teralogarítmica para el diseño de estructura con aislamiento sísmico, se deben definir a su vez los siguientes aspectos: (1) el modelo histerético de interés y sus parámetros, (2) una base representativa de registros de movimientos del terreno (acelerogramas) para una zona sísmica y tipo de terreno de interés y, (3) un criterio racional que permita definir las curvas de diseño. Estos tres aspectos deben ser valorados cuidadosamente si los EDC se pretenden desarrollar o asociar para reglamentos de diseño sísmico específicos. En el presente trabajo se definen EDC para aisladores de comportamiento bilineal asociados a movimientos del terreno representativos de suelos firmes (roca) registrados en la Costa mexicana del Pacífico durante sismos recientes de gran magnitud, utilizando un criterio estadístico simple. Estos EDC se definieron para evaluar su efectividad para el diseño de estructuras con aisladores de base de este tipo, pero no se han desarrollado aún para satisfacer los criterios empleados en los reglamentos de México para la costa mexicana del Pacífico, como son el Manual de Obras Civiles o el Reglamento de Construcciones del Estado de Guerrero. Los criterios específicos considerados para los puntos 1 a 3 señalados líneas atrás se describen brevemente a continuación.

\section{Modelo histerético seleccionado}

Se utilizó un modelo histerético bilineal (fig 4) con una pendiente posterior a la fluencia del $10 \%$ de la rigidez elástica inicial del sistema $\left(\mathrm{k}_{2} / \mathrm{k}_{1}=0.10\right)$, ya que es una relación típica para la mayoría de los elastómeros laminados (con y sin corazón de plomo, de alto amortiguamiento) que se comercian actualmente. Se seleccionaron dos relaciones de cortante de fluencia que cubrieran un intervalo práctico de aplicación ante la acción de sismos de gran magnitud: $\mathrm{V}_{\mathrm{y}} / \mathrm{W}=0.05 \mathrm{y}$ $\mathrm{V}_{\mathrm{y}} / \mathrm{W}=0.10$.

\section{Registros de aceleración seleccionados}

Se emplearon acelerogramas típicos de terrenos firmes registrados en la costa mexicana del Pacífico durante sismos recientes de gran magnitud. Se consideraron los siguientes eventos: (a) el sismo de Michoacán del 19 de septiembre de $1985\left(\mathrm{M}_{\mathrm{s}}=8.1\right)$, (b) la réplica del sismo de Michoacán del 20 de septiembre de $1985\left(\mathrm{M}_{\mathrm{s}}=7.6\right)$, (c) el sismo del 25 de abril de $1989\left(\mathrm{M}_{\mathrm{s}}=6.9\right)$ $\mathrm{y}$, (d) el sismo de Manzanillo del 9 de octubre de $1995\left(\mathrm{M}_{\mathrm{w}}=8.0\right)$. Se dispusieron de 42 acelerogramas de las componentes horizontales asociadas a 15 estaciones acelerográficas diferentes. Se decidió entonces seleccionar a estaciones típicas de terreno firme roca, sin efectos de sitio, con lo cual se descartaron dos estaciones (Zacatula y Termoeléctrica de Manzanillo). Además, como sólo se contaba con una estación (San Marcos) que era epicentral para el sismo del 25 de abril de 1989, se decidió también no tomarla en cuenta por tal motivo. Entonces, quedaron 12 estaciones costeras en roca y 34 acelerogramas después de este primer filtro, donde ya no se incluyó al sismo de Manzanillo pues el único registro con que se contaba era precisamente el de la Termoeléctrica. 
Los 34 acelerogramas restantes se escalaron de manera que todos tuvieran la misma aceleración espectral que el espectro de diseño de la zona D-I propuesto por el Manual de Obras Civiles de 1993 (MOC-93) para un periodo $\mathrm{T}=2.24 \mathrm{~s}$, como se ilustra para algunos registros en la figura 6. Se seleccionó $\mathrm{T}=2.24 \mathrm{~s}$ porque es un valor intermedio en el intervalo de periodos $1.5 \mathrm{~s}<\mathrm{T}_{\mathrm{a}}<3.0 \mathrm{~s}$ considerado para el diseño sísmico de sistemas aislados utilizando el método estático por reglamentos como el UBC-97.

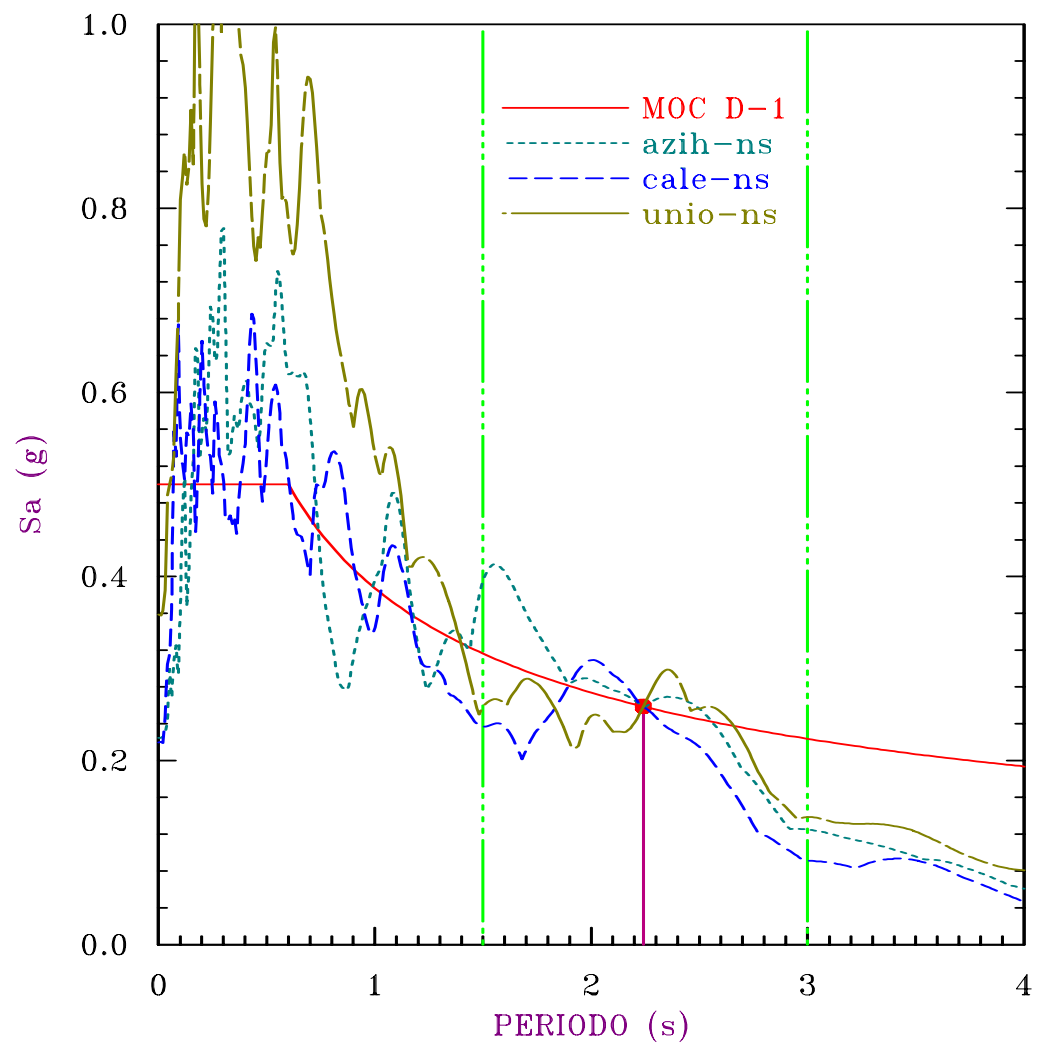

Figura 6. Escalamiento de algunos de los acelerogramas seleccionados de manera que su aceleración espectral para $\mathrm{T}=2.24 \mathrm{~s}$ coincida con la del espectro de diseño de la zona D-I del Manual de Obras Civiles (MOC) de Comisión Federal de Electricidad.

Dado que varios de los acelerogramas seleccionados requerían de un factor de amplificación muy grande, que en consecuencia amplificaban la aceleración máxima del terreno a valores totalmente fuera de la realidad, muy por encima de los valores de aceleraciones máximas del terreno jamás registradas en roca durante sismos intensos (por ejemplo, $a_{\max }=5.11 \mathrm{~g}$ para la estación VIGA del sismo del 25 de abril de 1989), se realizó un último filtrado donde se vigiló que los acelerogramas escalados utilizados no sobrepasaran aceleraciones máximas del terreno mayores a $1.2 \mathrm{~g}$, ni que el factor de escala utilizado pasara de 10.0, de donde quedaron los 16 acelerogramas correspondientes a las nueve estaciones acelerográficas que se indican en la tabla 1. La aceleración máxima del terreno promedio de estos 16 registros fue $\mathrm{a}_{\max }=0.558 \mathrm{~g}$. 
Tabla 1. Características de los acelerogramas seleccionados

\begin{tabular}{|c|c|c|c|c|c|c|c|}
\hline \multirow[t]{2}{*}{ Evento } & \multirow[t]{2}{*}{ Estación } & \multicolumn{2}{|c|}{ Duración (s) } & \multicolumn{2}{|c|}{$\begin{array}{l}\mathrm{A}_{\max } \\
\left(\mathrm{cm} / \mathrm{s}^{2}\right)\end{array}$} & \multicolumn{2}{|c|}{$\begin{array}{c}\text { Factor de escala }(f) \text { para igualar la amplitud } \\
\text { del espectro de la zona D-I para } \mathrm{T}=2.24 \mathrm{~s}\end{array}$} \\
\hline & & Registro & $\begin{array}{c}\text { Fase } \\
\text { intensa }\end{array}$ & E-W & $\mathrm{N}-\mathrm{S}$ & E-W & $\mathrm{N}-\mathrm{S}$ \\
\hline \multirow[t]{7}{*}{$09 / 19 / 85$} & AZIH & 71.8 & 20 & 162. & 101 & 2.241 & 2.141 \\
\hline & CALE & 48.9 & 10 & 138. & 138. & 2.468 & 1.570 \\
\hline & PAPN & 59.1 & 5 & 114. & 157. & $12.909 *$ & 7.282 \\
\hline & PARS & 52.6 & 16 & 89. & 116. & $13.041 *$ & 6.722 \\
\hline & SUCH & 59.6 & 5 & 81. & 102. & 7.224 & 4.989 \\
\hline & UNIO & 62.7 & 25 & 147. & 163. & 3.695 & 2.124 \\
\hline & VILE & 63.6 & 7 & 120 & 125 & 4.369 & 3.668 \\
\hline $20 / 09 / 85$ & AZIH & 54.0 & 15 & 153. & 134. & 3.154 & 4.543 \\
\hline $04 / 25 / 89$ & CPDR & 37.8 & 5 & 93. & 103. & 7.642 & 8.492 \\
\hline
\end{tabular}

\section{Criterio para definir las curvas de diseño}

El criterio empleado para definir las curvas de diseño para los EDC estuvo definido tanto por la selección y criterio de escalamiento de los movimientos del terreno como por el objetivo principal del estudio, que es evaluar la efectividad y el potencial de los EDC para el diseño de estructuras con aislamiento sísmico, más que definir EDC asociados a un reglamento en particular. La definición de EDC para reglamentos específicos requiere de análisis completos de peligro sísmico donde se deben seguir pasos muy rigurosos en la selección de los movimientos del terreno, definir magnitudes (tamaños) de los sismos de diseño para distintos niveles de desempeño, fijar tasas de excedencia, etc. Con base en lo expuesto anteriormente, se decidió definir los espectros de diseño por capacidad en función del espectro correspondiente a la media más una vez la desviación estándar $\left(\sigma+S_{\text {dev }}\right)$ de los 16 registros acelerográficos escalados e identificados en la tabla 1. Este criterio está asociado a una función acumulada de probabilidad de $84.13 \%$ para la distribución normal típica, y se considera un criterio razonable, aunque no conservador, dado lo reducido de la muestra seleccionada por las razones que se expusieron en secciones anteriores. 


\section{Procedimiento para definir EDC específicos}

Con fines ilustrativos, en esta sección se presenta el procedimiento utilizado por el autor para definir EDC para aisladores de comportamiento bilineal con $\mathrm{V}_{\mathrm{y}} / \mathrm{W}=0.10$ y k $\mathrm{k}_{2} / \mathrm{k}_{1}=0.10$ (figura 7). El procedimiento sigue los lineamientos y criterios generales descritos en secciones anteriores y fue utilizado también para cuando $\mathrm{V}_{\mathrm{y}} / \mathrm{W}=0.05$ (fig 8). Los espectros de capacidad correspondientes a la media más una vez la desviación estándar $\left(\sigma+\mathrm{S}_{\mathrm{dev}}\right)$ cuando se consideran los 16 registros acelerográficos se presentan también en las figuras 7 y 8 , donde se ilustra en particular el espectro de diseño por desplazamiento (EDD), que es el más importante para el diseño del sistema de aislamiento.

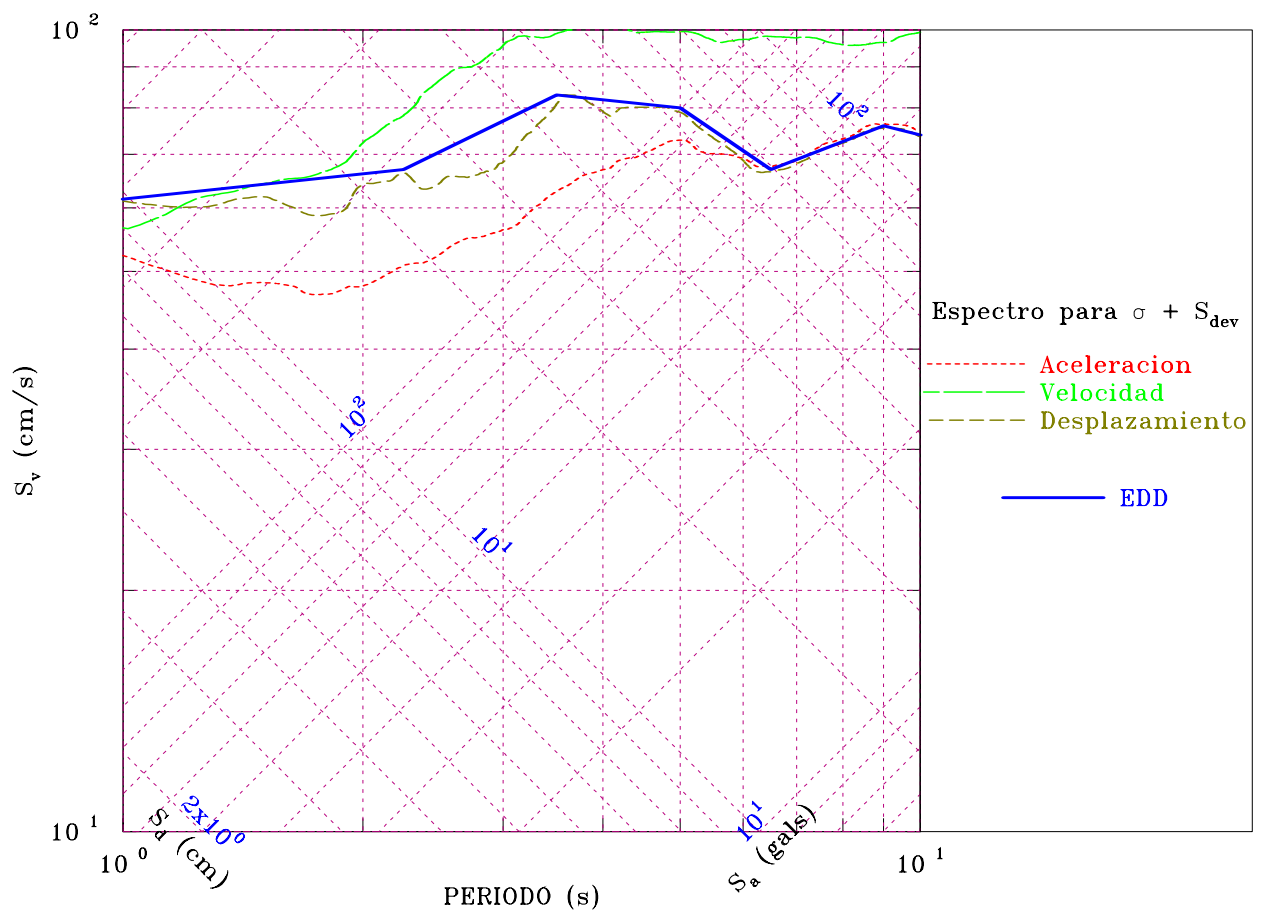

Figura 7. EDC para aisladores de comportamiento bilineal con parámetros $\mathrm{V}_{\mathrm{y}} / \mathrm{W}=0.10 \mathrm{y}$

$$
\mathrm{k}_{2} / \mathrm{k}_{1}=0.10
$$

Tomando en cuenta que:

(a) el aislamiento sísmico de estructuras es una solución estructural eficiente para periodos efectivos $\mathrm{T}_{\mathrm{a}}$ mayores o iguales a 1.5 segundos,

(b) reglamentos con criterios de diseño sísmico para aisladores de base, como el reglamento norteamericano UBC, establecen límites recomendados entre $1.5 \mathrm{~s}$ y $3 \mathrm{~s}\left(1.5 \mathrm{~s} \leq \mathrm{T}_{\mathrm{a}} \leq 3 \mathrm{~s}\right)$ para utilizar los criterios de diseño estático y algunos de sus criterios de diseño dinámico,

(c) del conocimiento del autor, el máximo periodo efectivo de aislamiento utilizado hasta la fecha en una estructura real corresponde al diseño de la reparación del edificio del ayuntamiento de la ciudad de Los Ángeles, California, donde se utilizó un periodo efectivo de aislamiento alrededor de 4s (Youssef, 1996),

(d) el diseño del sistema de aislamiento está controlado por los desplazamientos máximos esperadas $y$, 
(e) el diseño de la superestructura está controlado por las aceleraciones máximas

se decidió entonces definir espectros envolventes de diseño para cada una de las dos respuestas del movimiento de interés (aceleración y desplazamiento) en el intervalo de periodos entre 1s y $4 \mathrm{~s}\left(1 \mathrm{~s} \leq \mathrm{T}_{\mathrm{a}} \leq 4 \mathrm{~s}\right)$ por lo menos, siendo menos conservadora la definición del espectro para periodos muy largos desde el punto de vista de estructuras convencionales $\left(\mathrm{T}_{\mathrm{a}}>6 \mathrm{~s}\right)$, como se ilustra en las figuras 7 y 8 en específico para el espectro de diseño por desplazamiento (EDD). Los espectros de diseño por aceleración (EDA) no se muestran en esta figura con la finalidad de no hacerla confusa, pero se definen de manera similar a los EDD, envolviendo al espectro de aceleraciones correspondiente a la media más una vez la desviación estándar.

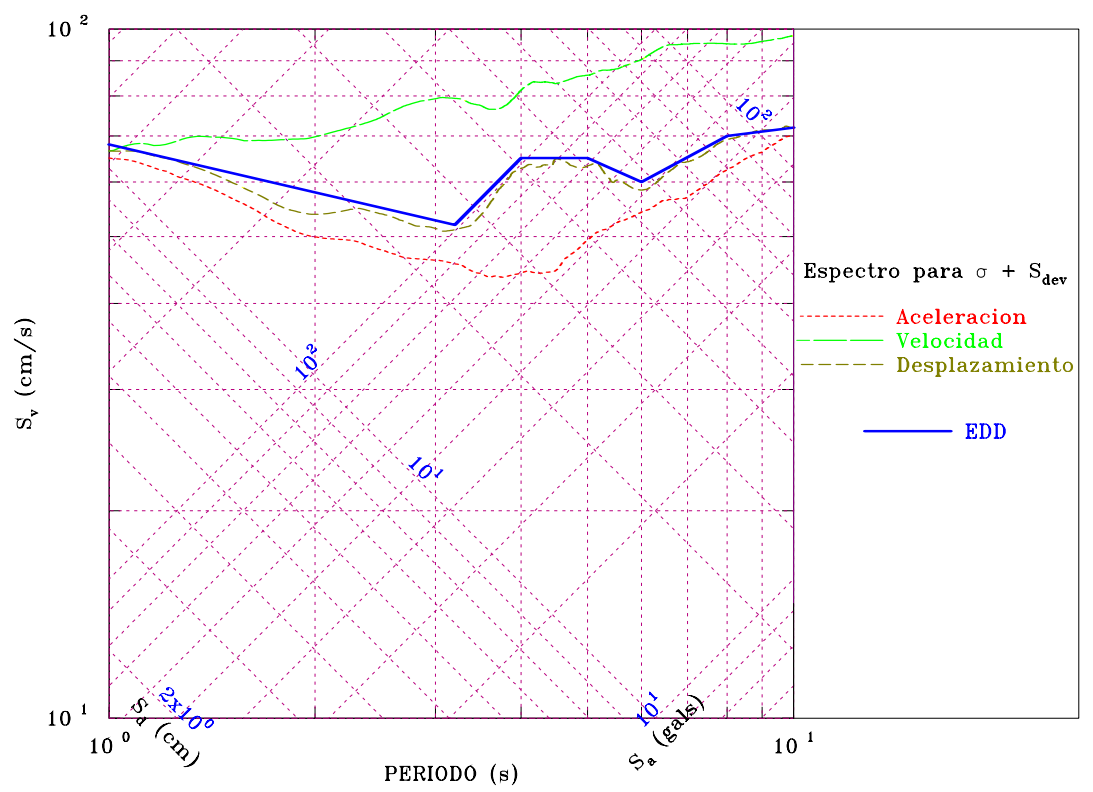

Figura 8. EDC para aisladores de comportamiento bilineal con parámetros $\mathrm{V}_{\mathrm{y}} / \mathrm{W}=0.05 \mathrm{y}$

$$
\mathrm{k}_{2} / \mathrm{k}_{1}=0.10
$$

Se intentó definir envolventes suaves que protejan de manera razonable el intervalo de periodos de mayor interés $\left(1.5 \mathrm{~s} \leq \mathrm{T}_{\mathrm{a}} \leq 3 \mathrm{~s}\right)$, como puede observarse de las figuras 7 y 8 , aunque con ello se sea conservador para algunos periodos comprendidos dentro del intervalo de interés.

\section{EDC propuestos}

Los espectros de diseño por desplazamiento (EDD) definidos en las figuras 7 y 8 se presentan y comparan en la figura 9. De la observación de los mismos podría pensarse que, para el intervalo de periodos de interés de los sistemas de aislamiento sísmico (1.5s $\left.\leq \mathrm{T}_{\mathrm{a}} \leq 3 \mathrm{~s}\right)$, resultaría más conveniente intentar diseñar para cortantes de cedencia menores $\left(\mathrm{V}_{\mathrm{y}} / \mathrm{W}=0.05\right)$, ya que los desplazamientos máximos son menores para esta envolvente. Sin embargo, esta observación es incompleta si uno no observa al mismo tiempo las curvas asociadas a las aceleraciones máximas (figs 7y 8), del cortante que se transmite a la superestructura (fig 11) y las demandas de ductilidad asociadas a estos espectros para $\sigma+\mathrm{S}_{\mathrm{dev}}$ (fig 10). 


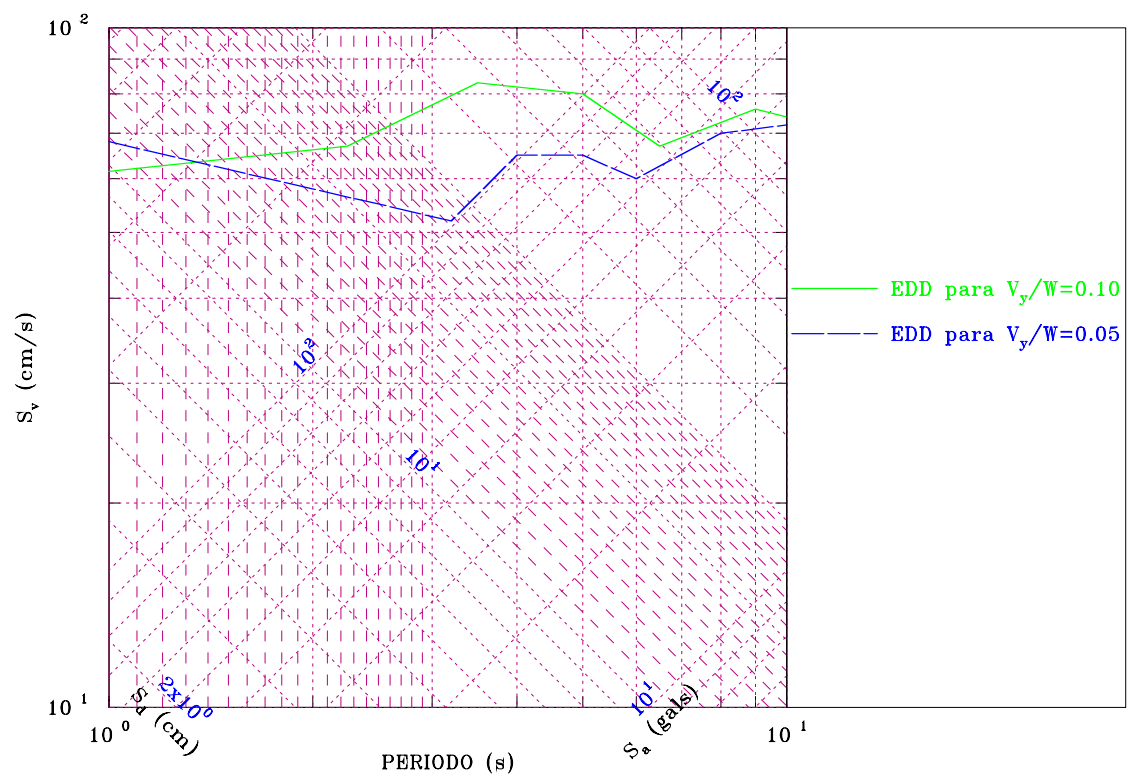

Figura 9. Espectros de diseño por desplazamiento (EDD) para aisladores bilineales con

$$
\mathrm{k}_{2} / \mathrm{k}_{1}=0.10
$$

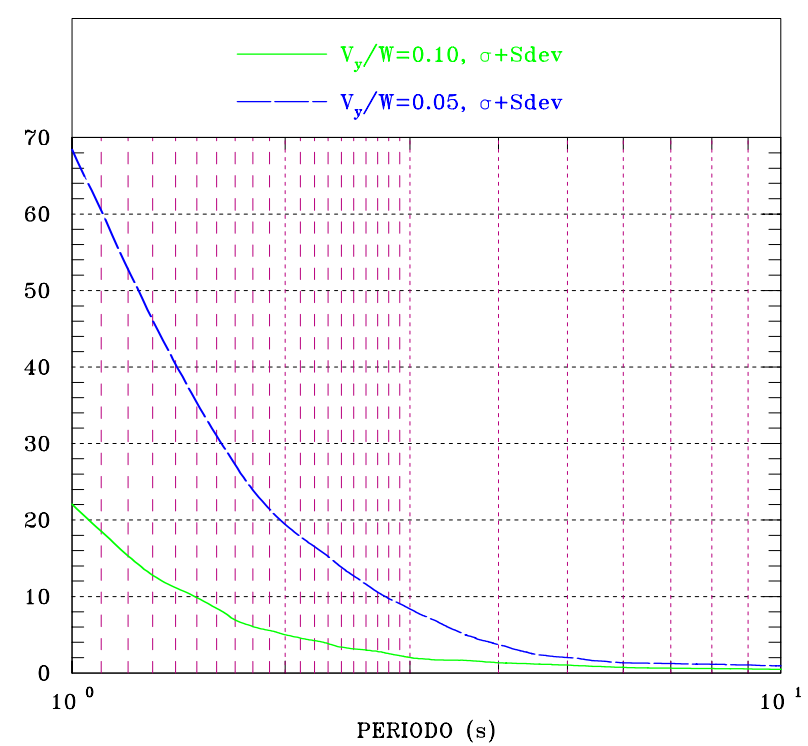

Figura 10. Demandas de ductilidad $(\mu)$ asociados a los espectros para $\sigma+S_{\text {dev }}$ presentados en las figuras 7 y 8 .

De la figura 10 se puede observar que las demandas de ductilidad asociadas a los espectros para $\mathrm{V}_{\mathrm{y}} / \mathrm{W}=0.05$ son muy altas para el intervalo de periodo de interés y que se encuentran en valores más razonables en ese mismo intervalo para $\mathrm{V}_{\mathrm{y}} / \mathrm{W}=0.10$. De $\operatorname{los}$ espectros de diseño para la superestructura (EDA) mostrados en la figura 11 se aprecia que para periodos entre $1.5 \mathrm{~s}$ y $2.0 \mathrm{~s}$ se transmitirían mayores cortantes a la superestructura para $\mathrm{V}_{\mathrm{y}} / \mathrm{W}=0.05$ y que a partir de un periodo de $2.2 \mathrm{~s}$ se transmitirían mayores cortantes para $\mathrm{V}_{\mathrm{y}} / \mathrm{W}=0.10$, por lo que se puede concluir que la selección del espectro más adecuado para el diseño de una estructura aislada específica es complicada, pues depende de muchos factores, por lo que no es adecuado generalizar en este sentido. 


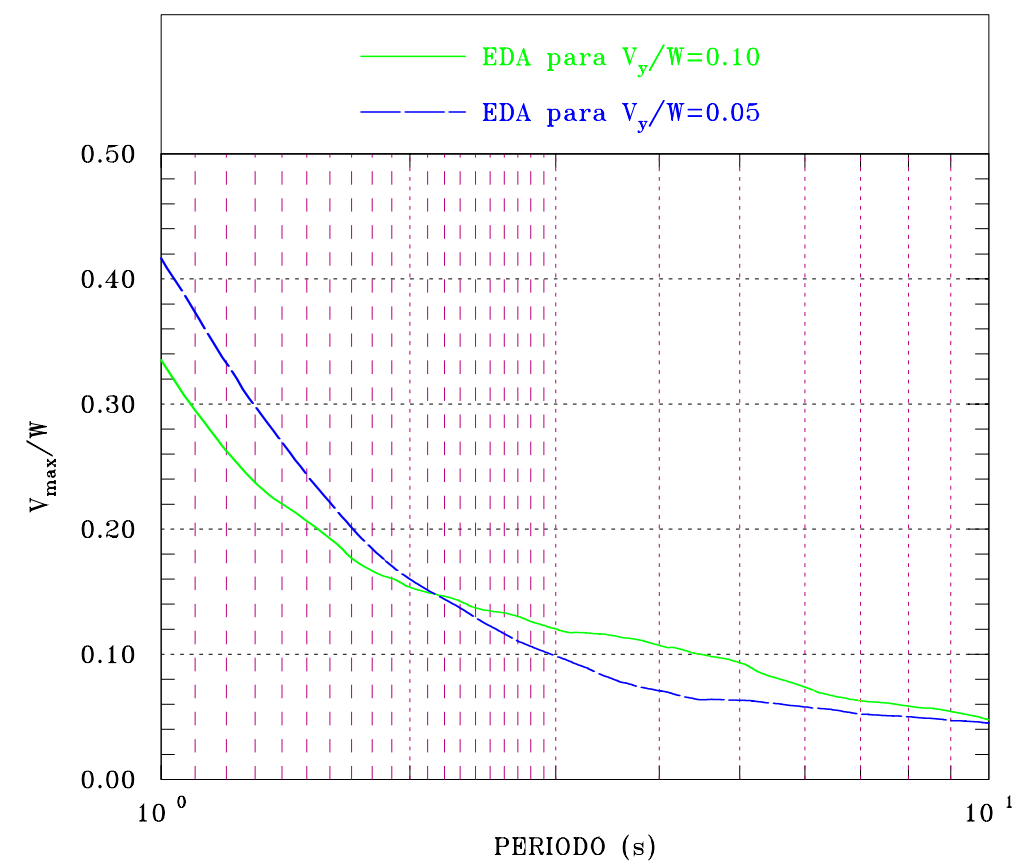

Figura 11. Espectro de diseño de aceleraciones (EDA) para el diseño de la superestructura de estructuras con aisladores bilineales con $\mathrm{k}_{2} / \mathrm{k}_{1}=0.10$

La curva de aceleraciones máximas es proporcional (y casi igual) a la curva de resistencias máximas normalizadas $\left(\mathrm{V}_{\max } / \mathrm{W}\right)$. Es, por tanto, conveniente trazar curvas independientes para definir el cortante máximo que se transmite a la superestructura. Aunque esto se puede hacer directamente en la gráfica tetralogarítmica, se decidió trazarlo en una gráfica independiente para facilitar su entendimiento. Las curvas de diseño para la superestructura, que esencialmente son los espectros de diseño de aceleraciones (EDA) se presentan en la figura 11. Se aprecia de las mismas que a medida que se aumenta el periodo disminuye el cortante máximo transmitido a la superestructura, lo que indica que el comportamiento no lineal de estructuras con periodos largos es más reducido cuando se sujetan a movimientos típicos de terrenos firmes. De estas gráficas se puede inferir a partir de qué periodos se espera que la respuesta sea totalmente elástica (aquéllos periodos donde $\mathrm{V}_{\max } / \mathrm{W}$ de la superestructura es menor al $\mathrm{V}_{\mathrm{y}} / \mathrm{W}$ de fluencia del aislador).

\section{PROCEDIMIENTO DE DISEÑO SUGERIDO}

El procedimiento sugerido para el diseño de los aisladores de base utilizando los espectros de diseño por capacidad (EDC) por desplazamiento (EDD) es el siguiente:

(1) Se selecciona $T_{a}$, tomando en cuenta que

$$
\begin{aligned}
& 1.5 \mathrm{~s} \leq T_{a} \leq 3 \mathrm{~s} \\
& T_{a} \geq 2 T
\end{aligned}
$$


(2) Se selecciona un cortante de fluencia inicial para el sistema de aislamiento $V_{y} / W=\beta$, intentando seleccionar el menor valor de $\beta$ disponible que satisfaga una demanda de ductilidad aceptable para el sistema de aislamiento y que se transmita el menor cortante posible a la superestructura, para lo cual sería útil contar con el EDA (fig 11) y el de demandas de ductilidad asociadas (fig 10).

(3) Con $T_{a} \mathrm{y} \mathrm{V}_{\mathrm{y}} / \mathrm{W}$, se entra al espectro de diseño por desplazamiento (EDD, fig 9) y se obtiene el desplazamiento de diseño $D$, asociado a $\beta$.

(4) Se calcula la rigidez total efectiva del sistema de aislamiento:

$$
k_{\min }=\frac{4 \pi^{2} W}{g T_{a}^{2}}
$$

(5) Se propone el número de aisladores, $N_{\text {ais }}$

(6) Se calcula la rigidez efectiva de cada uno de los aisladores:

$$
k_{e f}=\frac{k_{\text {min }}}{N_{\text {ais }}}
$$

(7) Se calcula el cortante de fluencia de cada uno de los aisladores asociado al espectro no lineal de capacidad:

$$
V_{y}=\frac{\beta W}{N_{a i s}}
$$

(8) Se calcula el cortante último de cada aislador, $V_{u}$ :

$$
V_{u}=k_{e f} D
$$

(9) Para aisladores de comportamiento bilineal, es esencial controlar sus características mecánicas de manera que se garantice su estabilidad ante carga dinámica. En la figura 4 se esquematiza el criterio propuesto por el reglamento UBC, donde se establece que la rigidez efectiva del sistema de aislamiento para el desplazamiento de diseño $\left(k_{e f}\right)$ debe ser igual o mayor que una tercera parte de la rigidez efectiva al $20 \%$ del desplazamiento de diseño $\left(k_{e f 2}\right)$. En trabajos anteriores (por ejemplo, Tena 1997, Tena et al 1997), se ha demostrado que para aisladores con $\mathrm{k}_{2} / \mathrm{k}_{1}=0.10$, se debe cumplir que $\mathrm{k}_{1}=5 \mathrm{k}_{\text {ef }}$ para satisfacer la condición anteriormente expuesta conforme a la curva de la figura 4. Satisfacer esta condición involucra hacer el procedimiento iterativo, y si se quiere satisfacer al dedillo, puede resultar un poco engorroso y hasta difícil. De hecho, de la figura 4 se puede obtener que si:

$$
\begin{aligned}
& k_{e f} \geq \frac{1}{3} k_{e f 2} \\
& \frac{V_{u}}{D} \geq \frac{1}{3} \frac{V_{y}+k_{2}\left(0.2 D-\Delta_{y}\right)}{0.2 D}
\end{aligned}
$$




$$
V_{u} \geq \frac{V_{y}+k_{2}\left(0.2 D-\Delta_{y}\right)}{0.6}=\frac{V_{y}+k_{2}\left(0.2 D-\Delta_{y}\right)}{\alpha}
$$

Por tanto, se propone utilizar un procedimiento basado en el método de la bisección introduciendo una variable $\alpha$ (ecuación 11) que se encuentre dentro de los criterios de estabilidad de elastómeros y que además conduzca a satisfacer razonablemente la curva primaria propuesta por el UBC. Se propone que el valor de $\alpha$ esté entre $0.5 \leq \alpha \leq 0.6$ para garantizar que los sistemas de aislamiento sean razonablemente flexibles ante carga lateral. Los valores más adecuados de $\alpha$ son los del límite superior, pues es justo con el valor del límite superior con el que se obtiene el aislador más flexible que se permite y donde se cumple que $\mathrm{k}_{1}=5 \mathrm{k}_{\text {eff., }}$, como se puede deducir de la ecuación 12 que se presenta más adelante. Por tanto, se propone inicialmente $\alpha=0.6$.

(10) Se calculan la rigidez inicial y posterior a la fluencia del aislador

$$
\begin{aligned}
& k_{1}=12.5 k_{e f}(1-\alpha) \\
& k_{2}=0.1 k_{1}
\end{aligned}
$$

(11) Se calcula el desplazamiento de fluencia de los aisladores con características histeréticas bilineales:

$$
\Delta_{y}=\frac{V_{y}}{k_{1}}
$$

se debe observar que el desplazamiento de fluencia se encuentre entre estos límites para garantizar una demanda de ductilidad máxima que sea razonable:

$$
0.067 \mathrm{D} \leq \Delta_{y} \leq 0.111 \mathrm{D}
$$

(12) Se calcula el valor de $\alpha$ involucrado con el diseño calculado $\left(\alpha_{c}\right)$ :

$$
\alpha_{c}=\frac{V_{y}+k_{2}\left(0.2 D-\Delta_{y}\right)}{V_{u}}
$$

(13) Se evalúa el valor de $\alpha_{c}$. Si $\alpha_{c}>0.6$, es obvio que el método no va a converger en el intervalo sugerido, por lo que se detiene el diseño y la solución pudiera ser disminuir el periodo efectivo del sistema aislado propuesto inicialmente. Si $\alpha_{c}<0.5$, la solución puede o no converger en el intervalo considerado. Por lo tanto, sólo si en dos iteraciones consecutivas $\alpha_{c}<0.5$, se detiene el diseño y la solución pudiera ser aumentar el periodo efectivo del sistema aislado.

(14) Se compara $\alpha_{c}$ con $\alpha$, utilizando un criterio racional de convergencia, por ejemplo:

$$
\left|\frac{\alpha_{c}}{\alpha}-1\right| \leq 0.01
$$

Si se cumple esta condición, se toma como bueno el diseño y se continúa el procedimiento de diseño. En caso contrario, se propone $\alpha=\alpha_{c}$ y se repite el procedimiento desde el paso (10). 
(15) Se dimensionan los aisladores y se revisan sus dimensiones para asegurar que el diseño sea adecuado. Por ejemplo, se debe revisar en aisladores de tipo elastomérico que éstos no sean muy cortos, ni aún peor, muy esbeltos. Los criterios de diseño dependen del tipo de aislador, y se pueden utilizar desde criterios establecidos en la literatura especializada (por ejemplo, Skinner et al 1993, Kelly 1993, Tena 1997, Tena et al 1997), como de ayudas de diseño del fabricante. En este trabajo se utilizan los criterios que ha venido utilizando el autor desde hace tiempo, y que se presentan en Tena (1997) y Tena et al (1997) y que se basan en los criterios de estabilidad dinámica expuestos por Skinner et al (1993). Para aisladores elastoméricos laminados de sección circular, se calcula el diámetro $\phi$ y su altura $h$ de la siguiente manera:

$$
\begin{aligned}
& \phi=3 D \\
& h=\frac{\pi \phi^{2} G_{a i s}}{4 k_{e f}}
\end{aligned}
$$

donde $G_{a i s}$ es el módulo de rigidez al corte del elastómero, mientras que para elastómeros laminados con corazón de plomo, el diámetro del elastómero se calcula de acuerdo con la ecuación 18, pero la altura se calcula en función de $k_{2}$ en lugar de $k_{e f}$, es decir:

$$
h=\frac{\pi \phi^{2} G_{a i s}}{4 k_{2}}
$$

en el caso de los elastómeros laminados con corazón de plomo, restaría dimensionar el corazón de plomo. El área $\left(A_{p b}\right)$ y el diámetro $\left(\phi_{p b}\right)$ requeridos se calculan de la siguiente manera:

$$
\begin{aligned}
A_{p b} & =\frac{V_{y}-k_{2} \Delta_{y}}{\tau_{p b}} \\
\phi_{p b} & =\sqrt{\frac{4 A_{p b}}{\pi}}
\end{aligned}
$$

donde $\tau_{\mathrm{pb}}$ es el esfuerzo cortante de diseño del plomo. En general, si los aisladores dan de dimensiones muy esbeltas, es porque se están proponiendo más aisladores que los que se requieren para el desplazamiento y periodo seleccionado en el diseño, por lo que la solución es proponer un menor número de aisladores hasta que se obtenga una relación de esbeltez óptima, como podrá deducirse de las ecuaciones 19 y 20, ya que $k_{e f}$ (ecuación 6) y $k_{2}$ (ecuación 11) dependen del número de aisladores seleccionados. En cambio, si los aisladores son muy cortos, la solución es proponer un mayor número de aisladores que los considerados inicialmente, hasta obtener una relación de esbeltez óptima.

\section{Efectos bidireccionales}

Los EDD de la figura 9 son envolventes de respuestas máximas unidireccionales, por lo que el desplazamiento de diseño $(D)$ tomado de esta gráfica corresponde realmente al máximo desplazamiento si la acción del sismo fuera unidireccional. 
En el diseño de cualquier estructura tridimensional (aislada sísmicamente o no) es muy importante tomar en cuenta la acción bidireccional de los movimientos del terreno. Ha sido costumbre de muchos reglamentos de diseño sísmico, entre ellos los reglamentos mexicanos, tomar en cuenta los efectos de ambos componentes horizontales del movimiento del terreno combinando, en cada dirección en que se analice la estructura, el 100 por ciento de los efectos del componente que obra en esa dirección y el 30 por ciento de los efectos del que obra perpendicularmente a ella, con los signos que resulten más desfavorables para cada concepto. Esta regla se basa en estudios que toman en cuenta las aceleraciones registradas y, como es obvio, la combinación es independiente del periodo fundamental de la estructura en cuestión. La extensión de esta regla al diseño por desplazamiento no está justificada en ningún estudio, sino más bien por la costumbre.

En el caso concreto del diseño de aisladores de base, el reglamento UBC especifica que para el diseño de aisladores basados en análisis dinámicos, se deben escalar los movimientos del terreno de manera que para el periodo de interés se obtenga 1.3 veces la ordenada espectral del espectro base de diseño establecido por el UBC para tomar en cuenta efectos bidireccionales (es decir una regla de 130\%), que como podrá razonarse, es una decisión reglamentaria arbitraria supuestamente basada en la regla del $100 \%+30 \%$, y que como comentan y demuestran Naeim y Kelly (1999), no son siquiera equivalentes. Para el diseño de aisladores conforme al método estático del reglamento UBC, es práctica común que los diseñadores de los Estados Unidos tomen la regla del 100\%+30\% para obtener el desplazamiento de diseño, aunque en el UBC no establece en el capítulo de las normas de diseño de aisladores que así deba hacerse conforme al método estático, pero se supone que así debe ser conforme a lo establecido a las normas para diseño por sismo y de la lectura del escalamiento de la ordenada espectral del espectro de diseño para análisis dinámicos.

Las interrogantes son si realmente esta regla para combinar efectos bidireccionales es: (1) aplicable también al diseño por desplazamiento, (2) independiente del periodo de la estructura y de las características de los movimientos del terreno y, (3) segura. Gómez y Tena (2000) presentan estudios pioneros en este sentido, donde se calcularon los cocientes entre los máximos desplazamientos dinámicos de aisladores de sistemas totalmente simétricos ante acción bidireccional con respecto a la acción unidireccional para sistemas de aislamiento con cortantes de fluencia $\mathrm{V}_{\mathrm{y}} / \mathrm{W}=0.05$ y $\mathrm{V}_{\mathrm{y}} / \mathrm{W}=0.10$. En la figura 12 se presenta sólo el caso donde se normalizan los desplazamientos bidireccionales con respecto a los unidireccionales para la componente más intensa de los acelerogramas de las estaciones La Unión (UNIO, roca), San Marcos (SMRZ, epicentral) y Termoeléctrica de Manzanillo (TMANZ, efectos de sitio) de los sismos referidos anteriormente. De la observación de la figura 12 se puede concluir, entre otras cosas, que los factores de amplificación resultado del cociente entre los desplazamientos máximos de los aisladores ante excitación bidireccional con respecto a los de excitación unidireccional: (1) no son constantes para el intervalo de periodos considerado, es decir, no son independientes del periodo del sistema aislado, (2) sus características parecen depender también de las características de los movimientos del terreno, (3) dependen de la fuerza de cedencia del sistema de aislamiento, (4) que pueden obtenerse amplificaciones mayores al 30\%. 


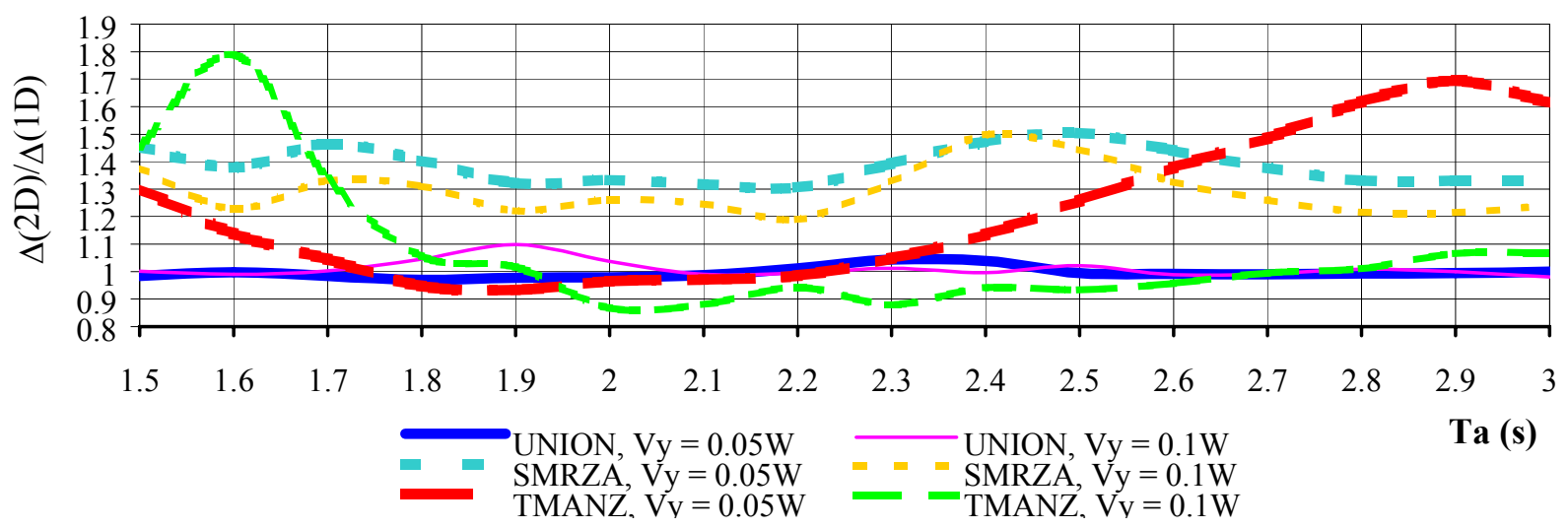

Figura 12. Relación entre el periodo aislado (Ta) y los máximos desplazamientos ante acción bidireccional normalizados con los máximos correspondientes a excitación N-S.

Es claro que del estudio de Gómez y Tena (2000) no se pueden especificar reglas de cómo deben combinarse acciones bidireccionales para determinar el desplazamiento de diseño del sistema de aislamiento, pero si da nueva luz en este sentido e indica que deben hacerse estudios paramétricos que consideren un mayor número de registros de características análogas (por ejemplo, típicos de roca) para estudiar las amplificaciones medias y sus respectivas desviaciones, con la finalidad de ver si pueden obtenerse expresiones simples que permitan llegar a propuestas más racionales para tomar en cuenta los efectos bidireccionales en el diseño de aisladores.

\section{DISEÑO DE ESTRUCTURAS AISLADAS UTILIZANDO EDC}

Para evaluar el diseño de sistemas de aislamiento de base utilizando los espectros de diseño por capacidad (EDC), se seleccionaron dos estructuras, una escuela pública de cuatro pisos que ha sido evaluada previamente por el autor y sus colaboradores utilizando otros procedimientos de diseño (por ejemplo, Tena-Colunga et al 1997, Tena 1997) y un edificio irregular de concreto reforzado de ocho pisos. Para ambas estructuras se utilizó una fuerza de fluencia normalizada para el sistema de aislamiento $\mathrm{V}_{\mathrm{y}} / \mathrm{W}=0.10$, y los detalles de estos diseños se discuten a continuación.

\section{Escuela EP2}

Es una escuela pública cuyo cuerpo principal consta de cuatro niveles (12.40 $\mathrm{m}$ de altura). La planta tipo del edificio y sus elevaciones se presentan en la figura 13. La orientación de la estructura es N-S en la dirección corta. El plantel consta de doce marcos que corren en dirección $\mathrm{N}-\mathrm{S}$ y dos marcos en dirección E-W. Sus dimensiones en planta son 42 x $8.7 \mathrm{~m}$. La estructura original consiste de marcos de concreto reforzado que en su dirección larga disponen de muros de mampostería de ladrillo hueco, con aberturas para ventanas y puertas, que se encuentran desligados de los marcos por medio de placas metálicas; mientras que en su dirección corta cuenta con muros estructurales de bloque de concreto no reforzados cada dos crujías. Los muros de los marcos exteriores 2 y 14 son muros de cortante de concreto reforzado. Las columnas y las 
vigas son de sección rectangular; las columnas miden aproximadamente 25 x $50 \mathrm{~cm}$, y están orientadas en la dirección corta, mientras que las vigas son de 25 x $50 \mathrm{~cm}$. El peralte de las losas es de $10 \mathrm{~cm}$. El refuerzo en columnas y trabes, y las propiedades del concreto y el acero de refuerzo corresponde a los diseños típicos de las escuelas del CAPFCE, y las propiedades de los muros de mampostería se tomaron de acuerdo con los valores recomendados por las Normas Técnicas Complementarias para Diseño de Estructuras de Mampostería del Reglamento de Construcciones para el Distrito Federal, como se discute con mayor detalle en otros trabajos (por ejemplo, Tena 1997 o Tena et al 1997). La estructura se modeló tridimensionalmente con el programa ETABS, y los detalles del modelo se discuten en Tena et al (1997), y las características dinámicas de sus modos fundamentales de vibración en cada dirección se resumen en la tabla 2.

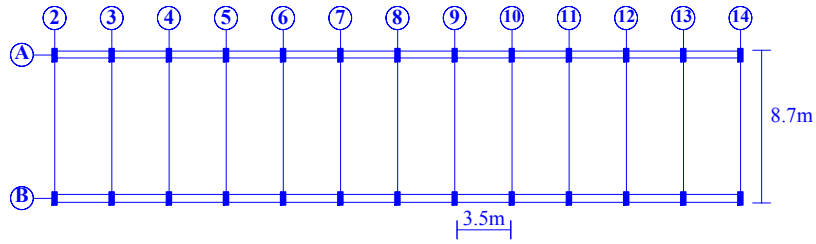

a) Planta

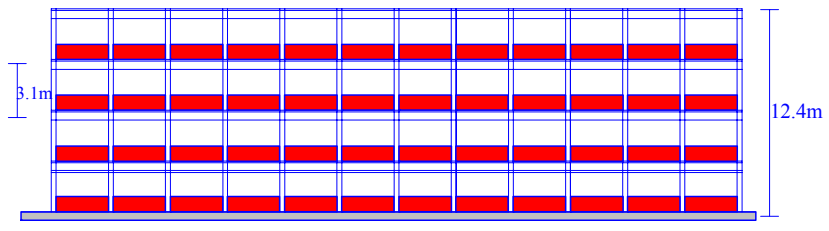

b) Elevación eje A

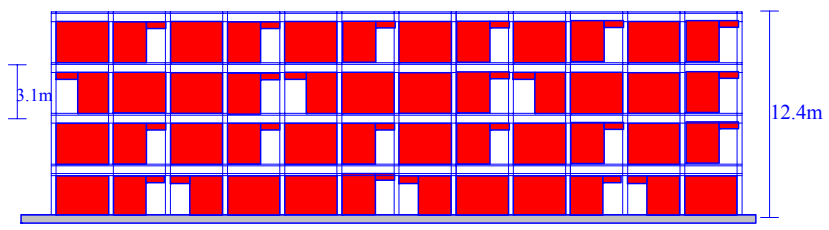

c) Elevación eje B

Figura 13. Planta y elevaciones de la escuela EP2

Tabla 2. Características dinámicas principales de la escuela EP2

\begin{tabular}{||c||c|c||c|c|c||}
\hline \multirow{2}{*}{ Estructuración } & \multirow{2}{*}{ Dirección } & \multirow{2}{*}{\begin{tabular}{c} 
Periodo \\
\cline { 3 - 5 }
\end{tabular}} & & \multicolumn{3}{|c||}{ Masa Modal (\%) } \\
\hline \hline \multirow{2}{*}{$\begin{array}{l}\text { Original EP2 con } \\
\text { muros longitudinales }\end{array}$} & $\mathrm{E}-\mathrm{W}$ & 0.63 & 88.35 & 0.00 & 0.00 \\
\cline { 2 - 6 } & $\mathrm{N}-\mathrm{S}$ & 0.12 & 0.00 & 85.54 & 0.00 \\
\hline
\end{tabular}




\section{Edificio ECO1}

Se trata de un edificio irregular destinado para oficinas corporativas y que está estructurado a base de vigas y columnas que forman marcos ortogonales unidos mediante un diafragma rígido. La estructura es irregular en planta, como se muestra en la figura 14. Consta de cuatro marcos en la dirección longitudinal (E-W) y cuatro en la dirección transversal (N-S). Los niveles uno al ocho cuentan con una área en planta de $456.22 \mathrm{~m}^{2}$ y de $235.19 \mathrm{~m}^{2}$ en el nivel de helipuerto, donde se reduce una crujía en ambas direcciones (fig 14). La elevación del eje D de la estructura se muestra en la fig 14, donde se aprecia que la altura entre el nivel del terreno natural y el nivel de helipuerto es de $33.08 \mathrm{~m}$, con alturas típicas de entrepiso de $4.03 \mathrm{~m}$ del primero al séptimo entrepisos y de $4.90 \mathrm{~m}$ para el nivel de helipuerto. Además, en esta estructura se presenta irregularidad importante en la dirección longitudinal, donde se eliminan la mayoría de las vigas en la crujía ejes A y B del marco 1 en toda la elevación del edificio (fig 14). Esta irregularidad trae consigo excentricidades importantes de rigidez y masa en la estructura, presentándose torsiones que se deben de considerar en los análisis. Además, introduce problemas de esbeltez en los muros perpendiculares a los ejes A y B y concentraciones de esfuerzo en la conexión existente entre los muros y la viga de acoplamiento de la crujía A-B en el último nivel.

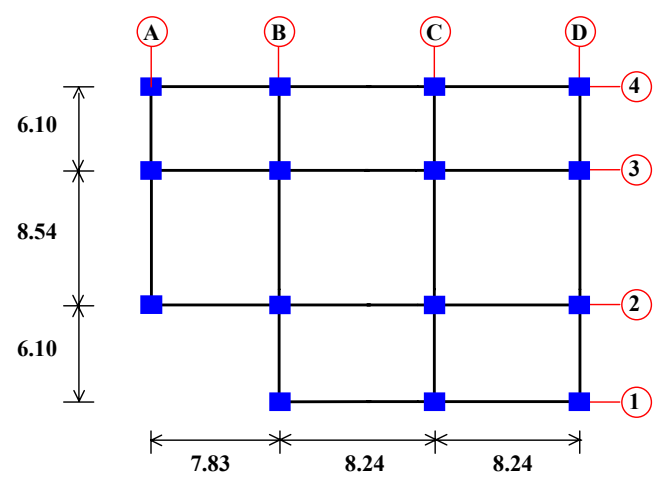

a) Planta típica

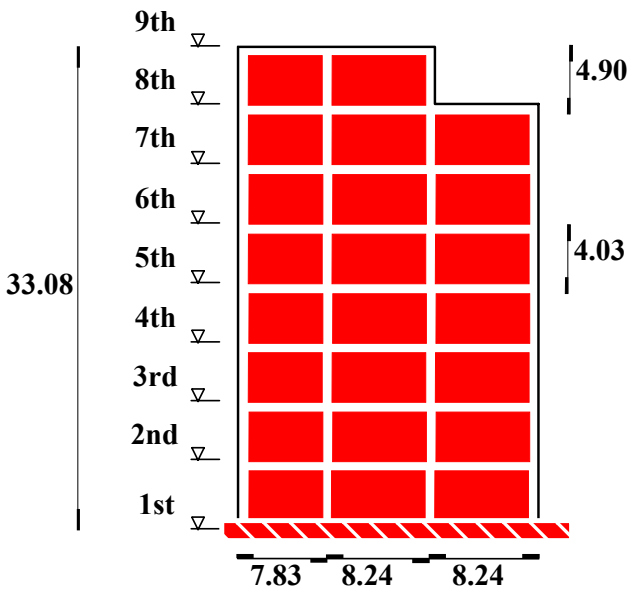

c) Elevación eje D

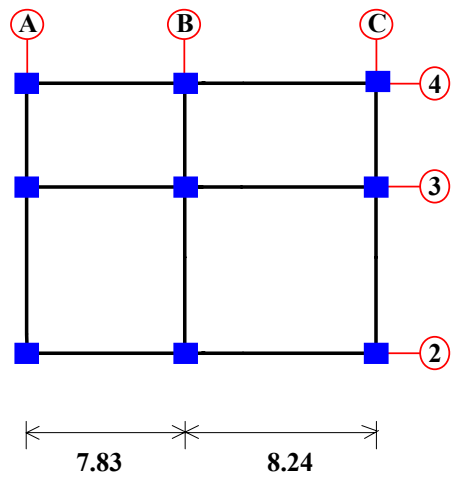

b) Planta del helipuerto

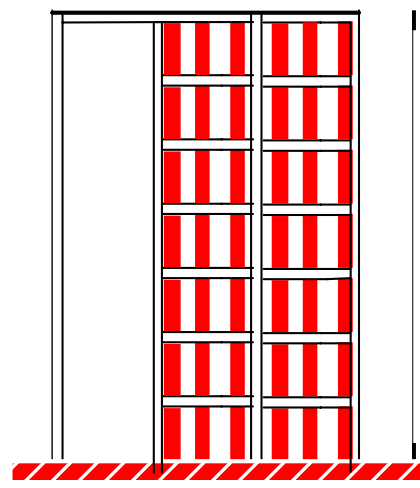

d) Elevación eje 1

Figura 14. Plantas y elevaciones del edificio ECO1 (dimensiones en metros) 
El sistema de piso es de losa plana aligerada cuyo peralte es de $25 \mathrm{~cm}$ con firme de compresión de $5 \mathrm{~cm}$ en todos los niveles. El ancho de las nervaduras es de $16 \mathrm{~cm}$ para las principales y de $12 \mathrm{~cm}$ para las secundarias, utilizando casetones formados con bloques de concreto ligero (cemento - arena) de 40 × 40 × $20 \mathrm{~cm}$ y 40 × 20 × $20 \mathrm{~cm}$. Las dimensiones de las columnas interiores y exteriores son $75 \times 75 \mathrm{~cm}$ y $70 \times 70 \mathrm{~cm}$ para el nivel dos, mientras que para los niveles superiores son de $60 \times 60 \mathrm{~cm}$. La resistencia a compresión simple del concreto en las columnas especificada en los planos originales es de $\mathrm{f}^{\prime} \mathrm{c}=250 \mathrm{~kg} / \mathrm{cm}^{2}$ para el nivel dos y 300 $\mathrm{kg} / \mathrm{cm}^{2}$ para los niveles superiores, con acero de refuerzo con $\mathrm{f}_{\mathrm{y}}=4200 \mathrm{~kg} / \mathrm{cm}^{2}$. El edificio también presenta muros estructurales perimetrales de concreto y de mampostería alrededor de la zona de elevadores, así como en las escaleras; el resto de los muros son divisorios de tabla-roca o cancelería que casi no aportan rigidez a la estructura, con un esfuerzo de compresión de la mampostería $\mathrm{f}^{*} \mathrm{~m}=15 \mathrm{~kg} / \mathrm{cm}^{2}$ para unidades de arcilla y de $20 \mathrm{~kg} / \mathrm{cm}^{2}$ para las de concreto. La cimentación del edificio está constituida por zapatas corridas con una profundidad de desplante de $1.5 \mathrm{~m}$ a partir del nivel del terreno natural.

Se construyó un modelo tridimensional de la estructura utilizando el programa de análisis estructural ETABS suponiendo que la estructura se encontraba empotrada en su base e incluyendo todos los modos representativos que permitieran garantizar que al menos el $90 \%$ de la masa modal total en cada grado de libertad global (dos de traslación y uno de rotación) participara en la respuesta dinámica. Los detalles del modelo se discuten en Tena et al (1997). Las características dinámicas de los modos fundamentales en cada dirección de esta estructura se resumen en la tabla 3 , de la cual se observa que a pesar de las irregularidades, los modos se encuentran solamente ligeramente acoplados.

Tabla 3. Características dinámicas principales del edificio ECO1

\begin{tabular}{||c||c||c||c|c|c||}
\hline \hline \multirow{2}{*}{ Estructuración } & \multirow{2}{*}{ Dirección } & \multirow{2}{*}{\begin{tabular}{c} 
Periodo \\
\cline { 3 - 5 }
\end{tabular}} & & \multicolumn{3}{|c||}{ Masa Modal (\%) } \\
\hline \hline \multirow{2}{*}{$\begin{array}{c}\text { ECO1 considerando } \\
\text { muros longitudinales }\end{array}$} & $\mathrm{E}-\mathrm{s})$ & 1.01 & 67.59 & 0.14 & 2.07 \\
\cline { 2 - 6 } & $\mathrm{N}-\mathrm{S}$ & 0.68 & 0.23 & 71.26 & 0.16 \\
\cline { 2 - 6 } & Rotación & 0.40 & 1.73 & 0.45 & 67.36 \\
\hline
\end{tabular}

\section{Diseño de los elastómeros}

Se diseñaron elastómeros laminados de alto amortiguamiento de las estructuras en estudio siguiendo el procedimiento sugerido anteriormente, en el cual el punto clave es la obtención del desplazamiento de diseño $D$ a partir de los EDD de la figura 9. 
Como se ha comentado anteriormente, para calibrar a los espectros de la fig 9 se debe considerar que la acción de los movimientos del terreno es unidireccional, por lo que en la tabla 4 se ilustra con detalle el proceso de diseño utilizado para los aisladores del plantel EP2 ante acción unidireccional conforme al procedimiento recomendado. Los diseños finales de EP2 y ECO1 ante acción unidireccional se resumen en la tabla 5.

También se decidió evaluar qué tan confiables son los diseños de sistemas de aislamiento utilizando la regla del 100\%+30\% para tomar en cuenta acciones bidireccionales. Los detalles del proceso de diseño utilizado para los aisladores del plantel EP2 ante acción bidireccional conforme al procedimiento recomendado se ilustra en la tabla 6 y los diseños finales de EP2 y ECO1 ante acción bidireccional se resumen en la tabla 7.

En el proceso de diseño se estudiaron distintos arreglos con diferente número de aisladores, hasta que se obtuvo un diseño satisfactorio para cada estructura. Cabe señalar, aunque pudiera ser obvio, que las dimensiones de los aisladores presentadas en las tablas 5 y 7 , que corresponden a un diseño preliminar, tendrían que adecuarse a dimensiones de aisladores comerciales para el diseño de una estructura real.

Tabla 4. Ilustración del proceso de diseño del sistema de aislamiento para la escuela EP2, considerando acción unidireccional, con base en el EDD de la figura 9

\begin{tabular}{|c|c|c|c|c|c|c|}
\hline \multicolumn{2}{|l|}{ Parámetros fijos } & Variables & Iteración 1 & Iteración 2 & Iteración 3 & Iteración 4 \\
\hline$T_{a}(\mathrm{~s})$ & 2.0 & $\alpha$ & 0.6 & 0.522 & 0.548 & 0.545 \\
\hline$W$ (Ton) & 1530 & $k_{l}(\mathrm{Ton} / \mathrm{cm})$ & 7.70 & 9.20 & 8.70 & 8.76 \\
\hline$\beta=V_{y} / W$ & 0.10 & $k_{2}(\mathrm{Ton} / \mathrm{cm})$ & 0.77 & 0.92 & 0.87 & 0.88 \\
\hline$D(\mathrm{~cm})$ & 21.2 & $\Delta_{y}(\mathrm{~cm})$ & 1.99 & 1.66 & 1.76 & 1.75 \\
\hline$N_{\text {ais }}$ & 10 & $\Delta_{y} / D$ & 0.094 & 0.078 & 0.083 & 0.082 \\
\hline$k_{\min }(\mathrm{Ton} / \mathrm{cm})$ & 15.39 & $\alpha_{c}$ & 0.522 & 0.548 & 0.545 & 0.545 \\
\hline$k_{e f}(\mathrm{Ton} / \mathrm{cm})$ & 1.54 & & & & & \\
\hline$V_{y}$ (Ton) & 15.30 & & & & & \\
\hline$V_{u}$ (Ton) & 32.63 & & & & & \\
\hline
\end{tabular}


Tabla 5. Resumen del diseño de aisladores y superestructura considerando acción unidireccional, con base en el EDD de la figura 9 y el EDA de la figura 11

\begin{tabular}{|c|c|c|c|c|c|}
\hline & EP2 & ECO1 & & EP2 & ECO1 \\
\hline \multicolumn{3}{|l|}{ Diseño global } & \multicolumn{3}{|c|}{ Características individuales de los aisladores } \\
\hline$T(\mathrm{~s})$ & 0.63 & 1.01 & $V u$ (Ton) & 32.63 & 54.01 \\
\hline$T_{a}(\mathrm{~s})$ & 2.0 & 2.5 & $V_{y}($ Ton $)$ & 15.30 & 29.96 \\
\hline$W$ (Ton) & 1530.0 & 4793.5 & $\Delta_{y}(\mathrm{~cm})$ & 1.75 & 3.11 \\
\hline$\beta=V_{y} / W$ & 0.10 & 0.10 & $\Delta_{M}=D(\mathrm{~cm})$ & 21.2 & 28.0 \\
\hline$D(\mathrm{~cm})$ (fig 9) & 21.2 & 28.0 & $k_{e f}(\mathrm{Ton} / \mathrm{cm})$ & 1.54 & 1.93 \\
\hline$N_{\text {ais }}$ & 10 & 16 & $k_{1}(\mathrm{Ton} / \mathrm{cm})$ & 8.76 & 9.65 \\
\hline$k_{\min }(\mathrm{Ton} / \mathrm{cm})$ & 15.39 & 30.865 & $k_{2}(\mathrm{Ton} / \mathrm{cm})$ & 0.88 & 0.97 \\
\hline$\alpha$ & 0.545 & 0.600 & $\Delta_{y} / D$ & 0.082 & 0.111 \\
\hline \multicolumn{3}{|c|}{ Parámetros para el diseño de la superestructura } & $\phi(\mathrm{cm})$ & 64 & 84 \\
\hline $\mathrm{V}_{\mathrm{S}} / \mathrm{W}($ fig 11$)$ & 0.154 & 0.135 & $h(\mathrm{~cm})$ & 21 & 30 \\
\hline$V_{S}$ (Ton) & 235.6 & 647.1 & $G_{\text {ais }}\left(\mathrm{kg} / \mathrm{cm}^{2}\right)$ & 10.2 & 10.2 \\
\hline
\end{tabular}

Tabla 6. Ilustración del proceso de diseño del sistema de aislamiento para la escuela EP2, considerando acción bidireccional, con base en el EDD de la figura 9

\begin{tabular}{|c|c|c|c|c|c|c|c|}
\hline & & & Itera. 1 & Itera. 2 & Itera. 3 & Itera. 4 & Itera. 5 \\
\hline$T_{a}(\mathrm{~s})$ & 2.5 & $\alpha$ & 0.6 & 0.484 & 0.513 & 0.506 & 0.508 \\
\hline$W$ (Ton) & 1530 & $k_{1}(\mathrm{Ton} / \mathrm{cm})$ & 8.21 & 10.59 & 9.99 & 10.14 & 10.10 \\
\hline$\beta=V_{y} / W$ & 0.10 & $k_{2}(\mathrm{Ton} / \mathrm{cm})$ & 0.82 & 1.06 & 1.00 & 1.01 & 1.01 \\
\hline$D$ (cm) (fig 9) & 28 & $\Delta_{y}(\mathrm{~cm})$ & 3.11 & 2.41 & 2.55 & 2.51 & 2.52 \\
\hline $\mathrm{D}_{2 \mathrm{D}}=\mathrm{D}+0.3 \mathrm{D}(\mathrm{cm})$ & 36.4 & $\Delta_{y} / D_{2 D}$ & 0.085 & 0.066 & 0.070 & 0.069 & 0.069 \\
\hline$N_{\text {ais }}$ & 6 & $\alpha_{c}$ & 0.484 & 0.513 & 0.506 & 0.508 & 0.507 \\
\hline$k_{\min }(\mathrm{Ton} / \mathrm{cm})$ & 9.85 & & & & & & \\
\hline$k_{e f}(\mathrm{Ton} / \mathrm{cm})$ & 1.64 & & & & & & \\
\hline$V_{y}$ (Ton) & 25.50 & & & & & & \\
\hline$V_{u}$ (Ton) & 59.76 & & & & & & \\
\hline
\end{tabular}


Tabla 7. Resumen del diseño de aisladores y superestructura considerando acción biidireccional, con base en el EDD de la figura 9 y el EDA de la figura 11

\begin{tabular}{|c|c|c|c|c|c|}
\hline & EP2 & ECO1 & & EP2 & ECO1 \\
\hline \multicolumn{3}{|l|}{ Diseño global } & \multicolumn{3}{|c|}{ Características individuales de los aisladores } \\
\hline$T(\mathrm{~s})$ & 0.63 & 1.01 & $V u$ (Ton) & 59.76 & 70.22 \\
\hline$T_{a}(\mathrm{~s})$ & 2.5 & 2.5 & $V_{y}($ Ton $)$ & 25.50 & 29.96 \\
\hline$W$ (Ton) & 1530.0 & 4793.5 & $\Delta_{y}(\mathrm{~cm})$ & 2.52 & 2.52 \\
\hline$\beta=V_{y} / W$ & 0.10 & 0.10 & $\Delta_{M}=D_{2 D}(\mathrm{~cm})$ & 36.4 & 36.4 \\
\hline$D(\mathrm{~cm})($ fig 9$)$ & 28.0 & 28.0 & $k_{e f}(\mathrm{Ton} / \mathrm{cm})$ & 1.64 & 1.93 \\
\hline $\mathrm{D}_{2 \mathrm{D}}=\mathrm{D}+0.3 \mathrm{D}(\mathrm{cm})$ & 36.4 & 36.4 & $k_{1}(\mathrm{Ton} / \mathrm{cm})$ & 10.10 & 11.86 \\
\hline$N_{\text {ais }}$ & 6 & 16 & $k_{2}(\mathrm{Ton} / \mathrm{cm})$ & 1.01 & 1.19 \\
\hline$k_{\min }(\mathrm{Ton} / \mathrm{cm})$ & 9.85 & 30.865 & $\Delta_{y} / D_{2 D}$ & 0.069 & 0.069 \\
\hline$\alpha$ & 0.508 & 0.508 & $\phi(\mathrm{cm})$ & 110 & 110 \\
\hline \multicolumn{3}{|c|}{ Parámetros para el diseño de la superestructura } & $h(\mathrm{~cm})$ & 58 & 50 \\
\hline $\mathrm{V}_{\mathrm{S}} / \mathrm{W}$ (fig 11) & 0.135 & 0.135 & $G_{\text {ais }}\left(\mathrm{kg} / \mathrm{cm}^{2}\right)$ & 10.2 & 10.2 \\
\hline $\mathrm{V}_{\mathrm{s} 2 \mathrm{D}} / \mathrm{W}=\mathrm{V}_{\mathrm{s}} / \mathrm{W}+0.3 \mathrm{~V}_{\mathrm{s}} / \mathrm{W}$ & 0.176 & 0.176 & & & \\
\hline$V_{s 2 D}$ (Ton) & 268.5 & 843.7 & & & \\
\hline
\end{tabular}

\section{Análisis dinámicos no lineales}

La efectividad de estos diseños se evaluó mediante análisis dinámicos no lineales utilizando el programa 3D-Basis (Nagarajaiah et al, 1991) ante los acelerogramas cuyas características se resumen en la tabla 1. Para los diseños basados en acción unidireccional (tabla 5) se consideró exclusivamente la acción unidireccional de cada acelerograma por separado, ya sea la componente E-W $(x)$ o N-S $(y)$, utilizando el factor de escala para cada componente del acelerograma según se especifica en esa misma tabla. Para los diseños que utilizan la regla del $100 \%+30 \%$ (tabla 7), se consideró la acción simultánea de las dos componentes acelerográficas horizontales de cada estación, pero en este caso se escalaron las dos componentes utilizando el factor de amplificación asociado a la componente más intensa, es decir, para el caso de la estación UNIO se utilizó $f=2.124$ asociado a la componente N-S, que es la más intensa para esta estación (tabla 1). Además, en el caso de la excitación bidireccional se estudió crudamente la incidencia de los movimientos del terreno al realizar análisis donde las componentes del terreno fueron intercambiadas, es decir, se definió como sismo " $x-y$ " cuando la componente E-W era paralela a la dirección $x$ de la estructura y la N-S paralela a la dirección $y$, mientras que un sismo " $y$ - $x$ " es cuando la componente E-W era paralela a la dirección $y$ y la N-S a la $x$. 
Los diseños deberían resultar totalmente satisfactorios ante todos los registros si se consideraran exclusivamente excitaciones unidireccionales, aunque dado lo reducido de la muestra y que se utilizó un criterio basado en la media más una desviación estándar, existe una cierta probabilidad de que los desplazamientos de diseño sean excedidos ya que la función acumulativa de probabilidad es del 84.13 (no tan cercano al 100\%). Ante la acción de componentes bidireccionales, siempre existe el riesgo de que los desplazamientos de diseño no cubran completamente las respuestas máximas utilizando la regla del $100 \%+30 \%$, pues como se ilustra en la figura 12, esta regla pudiera no ser conservadora para algunos registros en el intervalo de periodos de interés

Tabla 8. Respuestas dinámicas máximas para el proyecto de aislamiento de la escuela EP2, acción unidireccional $\left(\mathrm{T}_{\mathrm{a}}=2.0 \mathrm{~s}\right)$

\begin{tabular}{|c||c||c|c||c|c||c|c||c|c||}
\hline \hline Estación & Dir & $\begin{array}{c}\Delta_{\mathrm{xmax}} \\
(\mathrm{mm})\end{array}$ & $\Delta_{\mathrm{ymax}}(\mathrm{mm})$ & $\Delta_{\mathrm{a}}(\mathrm{cm})$ & $\Delta_{\mathrm{a}} / \Delta_{\mathrm{M}}$ & $\mathrm{V}_{\mathrm{xe}} / \mathrm{V}_{\mathrm{xa}}$ & $\mathrm{V}_{\mathrm{ye}} / \mathrm{V}_{\mathrm{ya}}$ & $\mathrm{V}_{\mathrm{xe}} / \mathrm{W}$ & $\mathrm{V}_{\mathrm{ye}} / \mathrm{W}$ \\
\hline \hline AZIH & $y$ & 19.0 & 0.1 & 12.62 & 0.60 & 0.83 & 0.71 & 0.135 & 0.003 \\
\hline CALE & $y$ & 14.8 & 0.0 & 11.42 & 0.54 & 0.73 & 0.75 & 0.114 & 0.001 \\
\hline UNIO & $y$ & 21.8 & 0.1 & 11.53 & 0.54 & 0.92 & 0.90 & 0.144 & 0.001 \\
\hline PAPN & $y$ & 21.7 & 0.1 & 20.30 & 0.96 & 0.81 & 0.86 & 0.168 & 0.001 \\
\hline VILE & $y$ & 31.4 & 0.2 & 20.49 & 0.97 & 0.99 & 0.73 & 0.176 & 0.000 \\
\hline SUCH & $y$ & 24.9 & 0.1 & 16.35 & 0.77 & 0.89 & 0.70 & 0.165 & 0.001 \\
\hline \hline
\end{tabular}

Los resultados de los análisis se resumen en las tablas 8 y 9 (EP2), 10 y 11 (ECO1). En ellas, $\Delta_{a}$ es el desplazamiento dinámico máximo del aislador en la dirección definida por $\theta$, que es el ángulo con que se presenta $\Delta_{a}$, medido con respecto al eje horizontal $(\mathrm{X}), \mathrm{D}_{\mathrm{xmax}} \mathrm{y} \mathrm{D}_{\mathrm{ymax}}$ son los desplazamientos relativos del último piso con respecto a la base en direcciones $x$ y $y$ respectivamente, $\mathrm{V}_{\mathrm{xe}} \mathrm{y} \mathrm{V}_{\mathrm{ye}}$ son los cortantes basales máximos transmitidos a la superestructura $\mathrm{y}$ $\mathrm{V}_{\mathrm{xa}} \mathrm{y} \mathrm{V}_{\text {ya }}$ son los cortantes máximos desarrollados en el sistema de aislamiento, y $\mathrm{W}$ es el peso de la estructura.

Tabla 9. Respuestas dinámicas máximas para el proyecto de aislamiento de la escuela EP2, acción bidireccional $\left(\mathrm{T}_{\mathrm{a}}=2.5 \mathrm{~s}\right)$

\begin{tabular}{|c||c||c|c|c|c|c|c||c|c||}
\hline \hline Estación & Dir & $\begin{array}{c}\Delta_{\text {xmax }} \\
(\mathrm{mm})\end{array}$ & $\Delta_{\text {ymax }}(\mathrm{mm})$ & $\Delta_{\mathrm{a}}(\mathrm{cm})$ & $\Delta_{\mathrm{a}} / \Delta_{\mathrm{M}}$ & $\mathrm{V}_{\mathrm{xe}} / \mathrm{V}_{\text {xa }}$ & $\mathrm{V}_{\text {ye }} / \mathrm{V}_{\text {ya }}$ & $\mathrm{V}_{\mathrm{xe}} / \mathrm{W}$ & $\mathrm{V}_{\text {ye }} / \mathrm{W}$ \\
\hline \hline AZIH & $y-x$ & 15.8 & 0.6 & 14.79 & 0.41 & 0.86 & 0.72 & 0.113 & 0.077 \\
\hline CALE & $y-x$ & 12.9 & 0.5 & 16.79 & 0.46 & 0.74 & 0.71 & 0.101 & 0.065 \\
\hline UNIO & $y-x$ & 16.7 & 0.6 & 14.34 & 0.39 & 0.84 & 0.71 & 0.112 & 0.078 \\
\hline PAPN & $x-y$ & 12.4 & 0.9 & 25.13 & 0.69 & 0.86 & 0.71 & 0.090 & 0.125 \\
\hline VILE & $y-x$ & 24.3 & 0.6 & 28.22 & 0.78 & 0.99 & 0.71 & 0.168 & 0.084 \\
\hline SUCH & $y-x$ & 20.0 & 0.6 & 17.37 & 0.48 & 0.90 & 0.71 & 0.134 & 0.079 \\
\hline \hline \multicolumn{8}{|c|}{$\Delta_{\mathrm{M}}=36.4 \mathrm{~cm}, \mathrm{~W}=1530$ Ton } \\
\hline
\end{tabular}


De las tablas 8 y 10 para acción unidireccional se observa que el diseño de los aisladores es satisfactorio para casi todas las estaciones, ya que la relación entre el desplazamiento máximo dinámico del aislador $\Delta_{a}$ es menor al desplazamiento máximo de diseño $\Delta_{M}$, por lo que su cociente resulta menor a la unidad, salvo para ECO1 y la estación PAPN, donde el desplazamiento es ligeramente superado $(6 \%)$, pero esto se puede asociar directamente a que utilizando una muestra reducida y un criterio estadístico como el de la media más una desviación estándar, existe una probabilidad no muy baja de que la respuesta máxima sea superada, por lo que se puede concluir que la confiabilidad de los espectros propuestos es aceptable conforme a los criterios estadísticos con los que fueron definidos. Es claro que se pueden obtener resultados "más seguros" utilizado un criterio más conservador asociado a un valor mayor de la función acumulativa de probabilidad (por ejemplo, la media más vez y media la desviación estándar). Por otra parte, de las tablas 9 y 11 se observa que, para estos casos específicos, la regla del $100 \%+30 \%$ resultó ser razonablemente segura para el diseño del sistema de aislamiento, ya que el cociente entre el desplazamiento máximo dinámico del aislador $\Delta_{a}$ entre al desplazamiento máximo de diseño $\Delta_{M}$ resulta ser siempre menor a la unidad.

Tabla 10. Respuestas dinámicas máximas para el proyecto de aislamiento del edificio ECO1, acción unidireccional $\left(\mathrm{T}_{\mathrm{a}}=2.5 \mathrm{~s}\right)$

\begin{tabular}{|c||c||c|c||c|c||c|c||c|c||}
\hline Estación & Dir & $\begin{array}{c}\Delta_{\text {xmax }} \\
(\mathrm{mm})\end{array}$ & $\begin{array}{c}\Delta_{\text {ymax }} \\
(\mathrm{mm})\end{array}$ & $\Delta_{\mathrm{a}}(\mathrm{cm})$ & $\Delta_{\mathrm{a}} / \Delta_{\mathrm{M}}$ & $\mathrm{V}_{\mathrm{xe}} / \mathrm{V}_{\mathrm{xa}}$ & $\mathrm{V}_{\text {ye }} / \mathrm{V}_{\text {ya }}$ & $\mathrm{V}_{\mathrm{xe}} / \mathrm{W}$ & $\mathrm{V}_{\mathrm{ye}} / \mathrm{W}$ \\
\hline \hline AZIH & $x$ & 42.9 & 5.0 & 15.92 & 0.57 & 0.89 & 1.07 & 0.103 & 0.018 \\
\hline CALE & $y$ & 50.5 & 5.3 & 17.39 & 0.62 & 0.89 & 0.92 & 0.105 & 0.017 \\
\hline UNIO & $y$ & 59.2 & 6.9 & 16.41 & 0.59 & 0.95 & 0.91 & 0.109 & 0.018 \\
\hline PAPN & $y$ & 71.7 & 8.9 & 29.57 & 1.06 & 0.78 & 1.03 & 0.120 & 0.015 \\
\hline VILE & $y$ & 69.0 & 8.4 & 23.14 & 0.83 & 0.90 & 0.97 & 0.137 & 0.020 \\
\hline SUCH & $y$ & 77.4 & 9.1 & 18.88 & 0.67 & 1.07 & 0.95 & 0.130 & 0.021 \\
\hline \hline
\end{tabular}

Tabla 11. Respuestas dinámicas máximas para el proyecto de aislamiento del edificio ECO1, acción bidireccional $\left(\mathrm{T}_{\mathrm{a}}=2.5 \mathrm{~s}\right)$

\begin{tabular}{|c||c||c|c||c|c||c|c||c|c||}
\hline \hline Estación & Dir & $\begin{array}{c}\Delta_{\text {xmax }} \\
(\mathrm{mm})\end{array}$ & $\begin{array}{c}\Delta_{\text {ymax }} \\
(\mathrm{mm})\end{array}$ & $\Delta_{\mathrm{a}}(\mathrm{cm})$ & $\Delta_{\mathrm{a}} / \Delta_{\mathrm{M}}$ & $\mathrm{V}_{\mathrm{xe}} / \mathrm{V}_{\text {xa }}$ & $\mathrm{V}_{\text {ye }} / \mathrm{V}_{\text {ya }}$ & $\mathrm{V}_{\text {xe }} / \mathrm{W}$ & $\mathrm{V}_{\text {ye }} / \mathrm{W}$ \\
\hline \hline AZIH & $x-y$ & 37.9 & 22.4 & 20.27 & 0.56 & 0.89 & 0.92 & 0.094 & 0.112 \\
\hline CALE & $y-x$ & 50.0 & 12.6 & 16.18 & 0.45 & 0.89 & 0.92 & 0.104 & 0.070 \\
\hline UNIO & $y-x$ & 62.9 & 19.3 & 16.88 & 0.46 & 1.00 & 0.86 & 0.110 & 0.088 \\
\hline PAPN & $y-x$ & 72.5 & 19.9 & 32.93 & 0.90 & 0.77 & 0.89 & 0.129 & 0.092 \\
\hline VILE & $y-x$ & 67.0 & 31.4 & 27.03 & 0.74 & 0.86 & 0.97 & 0.119 & 0.102 \\
\hline SUCH & $y-x$ & 73.9 & 18.4 & 20.44 & 0.56 & 1.01 & 0.93 & 0.134 & 0.085 \\
\hline \hline \multicolumn{10}{|c|}{$\Delta_{\mathrm{M}}=36.4 \mathrm{~cm}, \mathrm{~W}=4793.5$ Ton } \\
\hline
\end{tabular}


Una respuesta importante en el diseño de un sistema aislado es observar en cuanto se reduce la fuerza cortante que se transmite a la superestructura $\left(\mathrm{V}_{\mathrm{xe}}\right)$ como consecuencia del amortiguamiento adicional que proporciona el sistema de aislamiento en la interfase como consecuencia de disipar energía inelásticamente. Es bien sabido que en general el cortante transmitido a la superestructura $\left(\mathrm{V}_{\mathrm{xe}}\right)$ es inferior al que desarrolla el sistema de aislamiento $\left(\mathrm{V}_{\mathrm{xa}}\right)$, aunque existen ocasiones en que por características complejas de acoplamiento dinámico $\mathrm{V}_{\mathrm{xe}}$ puede resultar incluso superior a $\mathrm{V}_{\mathrm{xa}}$. Se observa de las tablas 8 a 11 que el cortante transmitido a la superestructura varía entre el 70\% y el 107\% del actuante en los aisladores, valores que se encuentran en el intervalo considerado por reglamentos de aislamiento sísmico, como el reglamento UBC, que considera valores entre $80 \%$ y $100 \%$. Para el plantel EP2, el cortante máximo transmitido a la superestructura estimado con el espectro de diseño (EDA) de la fig 11 $\left(\mathrm{V}_{\mathrm{S}} / \mathrm{W}=0.154\right.$, tabla 5$)$ es inferior a los cortantes dinámicos máximos ante acción unidireccional obtenidos para las estaciones VILE, PAPN y SUCH, pero superior para AZIH, UNIO y CALE $\left(\mathrm{V}_{\mathrm{xe}} / \mathrm{W}\right.$, tabla 8); sin embargo, para el edificio ECO1 y acción unidireccional (tabla 10), se puede observar que para la mayoría de los registros considerados, excepto la estación VILE, el cortante dinámico máximo es inferior al estimado en el diseño $\left(\mathrm{V}_{\mathrm{S}} / \mathrm{W}=0.135\right.$, tabla 5$)$, por lo que para la mayoría de los casos presentados la estimación del cortante transmitido a la superestructura a partir de espectros basados en la media más una desviación estándar resulta razonable, aunque no conservador. Para los diseños que utilizan la regla del 100\%+30\% (tablas 9 y 11), se aprecia que en ningún caso los cortantes dinámicos máximos transmitidos a la superestructura $\left(\mathrm{V}_{\mathrm{xe}} / \mathrm{W}\right)$ sobrepasan el estimado a partir de los espectros de diseño $\left(\mathrm{V}_{\mathrm{S}} / \mathrm{W}=0.176\right.$, tabla 7$)$, tanto para el plantel EP2 (tabla 9) como para el edificio ECO1 (tabla 11).

Para el edificio ECO1 se observa menor eficiencia en el sistema de aislamiento en reducir los cortantes basales que se transmiten a la superestructura, ya que en la mayoría de los registros el cortante transmitido a la superestructura oscila entre el $77 \%$ y el $100 \%$ del experimentado por el aislador, llegando a ser mayor en un 7\% para la estación $\mathrm{SUCH}$ en la dirección principal de análisis ante acción unidireccional (tabla 10).

Se puede concluir que el método de diseño empleado, utilizando espectros de diseño por capacidad, resultó en general satisfactorio a pesar de no contar con una base de datos más representativa y de no haber empleado un criterio estadístico conservador, al basarse los espectros propuesto en la media más una desviación estándar de las respuestas obtenidas.

\section{RESUMEN Y CONCLUSIONES}

Este trabajo presentó el concepto de espectros de diseño por capacidad (EDC) para estructuras aisladas sísmicamente (EDC), particularmente estructuras cuyo sistema de aislamiento tiene un comportamiento histerético bilineal. Los EDC relacionan respuestas máximas no lineales de aceleración, velocidad y desplazamiento con el periodo efectivo del sistema de aislamiento para sistemas bilineales definidos a partir de su fuerza de fluencia (o cedencia) y de su pendiente posterior a la fluencia. Estos espectros no lineales de capacidad pueden ser muy útiles para intentar llegar a diseños óptimos de sistemas de aislamiento sísmico con comportamiento bilineal, ya que en general, en la práctica mundial se intenta, en lo posible, diseñar a los sistemas de aislamiento para la menor fuerza de fluencia que brinde un nivel de seguridad razonable o 
requerido, ya que el costo de muchos sistemas de aislamiento se incrementa a medida que aumenta su fuerza de fluencia de diseño, particularmente para sistemas elastoméricos.

El concepto de EDC y su potencial aplicación se ilustró con el estudio de dos estructuras, donde el diseño del sistema de aislamiento se hizo utilizando los EDC y su eficacia se evaluó por medio de análisis dinámicos (paso a paso) no lineales de los modelos ante la acción de componentes unidireccionales y bidireccionales de los movimientos del terreno para una familia de acelerogramas asociados a los EDC. Los resultados de estos estudios sugieren que los EDC propuestos pueden constituir una herramienta útil y confiable para el diseño de estructuras aisladas sísmicamente con sistemas con comportamiento histerético bilineal, sin embargo, también muestra que antes de definir espectros con fines reglamentarios se deben realizar estudios más detallados y extensos donde se emplee una base de datos más representativa y emplear criterios más seguros para definir las curvas de diseño que basarse en un criterio simple (pero no conservador) como es utilizar la media más una desviación estándar de las respuestas máximas obtenidas. De realizarse estos estudios, permitirá en el futuro, llegar a diseños óptimos y confiables de aisladores de base con comportamiento bilineal, como es la familia de aisladores de base de tipo elastomérico. Por ello, se requieren continuar los esfuerzos de investigación que se han conducido en esta línea para definir EDC que puedan incorporarse en los reglamentos mexicanos de diseño sísmico.

\section{AGRADECIMIENTOS}

Esta investigación se inició en el Centro de Investigación Sísmica y ha sido posible continuarla gracias tanto al patrocinio interno de la UAM Azcapotzalco, como al del Consejo Nacional de Ciencia y Tecnología (CONACYT) como parte del proyecto Ref. 27738 U. El autor agradece los comentarios y sugerencias de Luis Esteva y de los revisores anónimos de este trabajo que han permitido enriquecerlo.

\section{REFERENCIAS}

Gómez Soberón, L. A. A. y A. Tena Colunga (2000), "Importancia de la excentricidad en la superestructura en la respuesta torsional de estructuras con aislamiento sísmico", Memorias, XII Congreso Nacional de Ingeniería Estructural, León, CDROM, noviembre.

Kelly, J M (1993), Earthquake-resistant design with rubber, primera edición, SpringerVerlang, Nueva York.

Kelly, J M (1999), Comunicación personal.

Mayes, R L (1997), Comunicación personal.

Miranda, E. (1993), "Evaluation of site-dependent inelastic seismic design spectra", ASCE Journal of Structural Engineering, Vol. 119, No. 5, mayo, pp. 1319-1338.

Naeim, F, y J M Kelly (1999), Design of seismic isolated structures, primera edición, John Wiley \& Sons, Nueva York. 
Nagarajaiah, S, A M Reinhorn y M C Constantinou (1991), "3D-Basis: Nonlinear dynamic analysis of three-dimensional base isolated structures: Part II," Technical Report NCEER-910005, National Center for Earthquake Engineering, State University of New York at Buffalo.

Newmark, N M y W J Hall (1982), Earthquake spectra and design, primera edición, Monograph Series, Earthquake Engineering Research Institute, Oakland.

Skinner, R I, W H Robinson y G H Mc Verry (1993), An introduction to seismic isolation, primera edición, John Wiley and Sons, Londres.

Tena-Colunga, A, C Gómez-Soberón y A Muñoz-Loustaunau (1997), "Seismic isolation of buildings subjected to typical subduction earthquake motions for the Mexican Pacific Coast", Earthquake Spectra, Vol. 13, No. 3, pp. 505-532.

Tena, A, L A A Gómez y M Salazar (1997), "Criterios de diseño y reglamentación de estructuras con aisladores de base para la costa del Pacífico," Reporte FJBS/CIS-97/17, Centro de Investigación Sísmica, AC, Fundación Javier Barros Sierra, diciembre.

Tena, A (1997), "Evaluación de un método de diseño estático para el aislamiento sísmico de estructuras de la costa Mexicana del Pacífico", Revista de Ingeniería Sísmica, SMIS, No. 57, pp 1-34, septiembre-diciembre.

"Uniform Building Code, 1997 Edition," (1997), International Conference of Building Officials, Whittier, California.

Villegas, O (1999), "Criterios de diseño dinámico para estructuras aisladas sísmicamente en las zonas costeras del Pacífico mexicano", Tesis de Maestría, División de Estudios de Posgrado de la Facultad de Ingeniería, Universidad Nacional Autónoma de México, febrero.

Villegas, O y A Tena (1999), "Criterios de diseño dinámico para estructuras aisladas sísmicamente en zonas costeras del Pacífico mexicano", Memorias, XII Congreso Nacional de Ingeniería Sísmica, Morelia, Michoacán, Vol. II, pp. 1172-1180, noviembre.

Villegas-Jiménez, O y A Tena-Colunga (2000), "Dynamic design procedure for the design of base isolated structures located on the Mexican Pacific Coast", Memorias, 12th World Conference on Earthquake Engineering, Auckland, Nueva Zelanda, Artículo No. 929, febrero.

Youssef, N (1996), "Application of hybrid damping system with base isolation for the seismic retrofit of Los Angeles Hall", Memorias, 11th World Conference on Earthquake Engineering, Acapulco, México, junio, Artículo No 2090. 